Heme and metformin coordinate human and murine macrophage heme oxygenase 1 expression with foam cell resistance partly via AMPK-ATF1

\author{
Xinyi Wan ${ }^{1}$ \\ Yilin Huo ${ }^{1}$ \\ Michael Johns ${ }^{1}$ \\ Emma Piper ${ }^{1}$ \\ Justin C Mason $^{1}$ \\ David Carling ${ }^{2}$ \\ Dorian O Haskard ${ }^{1}$ \\ Joseph J Boyle ${ }^{1^{*}}$
}

1. Vascular Sciences, NHLI, Imperial College London

2. MRC CSC, Imperial College London

*Corresponding author, Address: Vascular Sciences, NHLI, Imperial College London, Email: joseph.boyle@imperial.ac.uk 


\section{Abstract (229)}

Background: Intraplaque hemorrhage (IPH) is a key driver of the progression of atherosclerotic plaques. We recently characterised Mhem as a novel macrophage phenotype that limits the atherogenicity of IPH. Mhem are directed by activating transcription factor 1 (ATF1), which is activated by phosphorylation. A better understanding of the counteratherogenic ATF1-Mhem pathway may facilitate anti-atherosclerotic therapies.

Methods: We tested the hypothesis that heme in pathologically relevant concentrations activates the ATF1-Mhem pathway via 5'-AMP-activated protein kinase (AMPK) in primary human monocyte-derived macrophages and mouse bone marrow macrophages.

Results: We found that heme $(10 \mu \mathrm{M})$ activates AMPK, and downstream ATF1 mediated coinduction of HO-1 and LXR that characterise Mhem. Heme increased macrophage phosphoAMPK, phospho-ATF1 and its target genes, and these effects were inhibited by the AMPK antagonist dorsomorphin, or by AMPK-knockdown with siRNA. The AMPK-activating oral hypoglycemic agent metformin also induced and phosphorylated ATF1 at a clinically relevant concentration $(10 \mu \mathrm{M})$. Functional effects of heme and metformin were inhibited by AMPK-knockdown and included suppression of macrophage oxidative stress; increased cholesterol export; protection from foam cell formation; and suppression of macrophage inflammatory activation (HLA-DR expression).

Conclusions: Our data indicate that heme activates the ATF1 pathway in human macrophages via AMPK, and that a similar response occurs following treatment of cells with metformin. Our results suggest an in vitro mechanism that may explain the clinical evidence that metformin has vascular protective effects beyond its role in treating hyperglycemia. 


\section{Introduction}

\section{Intraplaque hemorrhage}

Intraplaque hemorrhage (IPH) drives atherosclerosis partly via the dual metabolic insult of cholesterol-enriched erythrocyte membranes and pro-oxidant heme/iron ${ }^{1}$. Indeed, IPH may acutely destabilise coronary lesions causing myocardial ischemia ${ }^{2}$. We have recently shown that IPH generates a distinct adaptive macrophage state (Mhem) in human plaques ${ }^{3}$. We went on to identify activating transcription factor 1 (ATF1) as the key transcription factor (TF) mediating adaptive responses to heme at $10 \mu \mathrm{M}$, a pathophysiologically relevant concentration $^{4}$. Others have independently designated a similar macrophage phenotype as $\mathrm{M}(\mathrm{Hb})^{5}$. Mhem exhibit coordinated properties including increased heme oxygenase (HO)-1 expression, reduced oxidative stress, suppressed inflammatory activation, increased lipid export and resistance to foam cell formation and increased levels of the anti-inflammatory cytokine interleukin-10 ${ }^{3}$. Taken together, these may inhibit atherogenesis if they also apply in vivo. Co-induction of the genes for HO-1 and LXR is a key component of ATF1 transcriptional network driving macrophages to become Mhem ${ }^{4}$.

\section{ATF1}

ATF1 is the closest homologue of cyclic-AMP (cAMP) response element binding protein 1 (CREB1). Like CREB1, ATF1 was named for its capacity to activate gene expression in response to cAMP ${ }^{7}$. ATF1 corresponds to a GWAS locus associated with sudden cardiac death in advanced coronary artery disease, consistent with a role in responding to IPH in vivo. Both CREB1 and ATF1 are phosphorylated on homologous motifs by cAMP-dependent protein kinase (protein kinase A, PKA) and 5'-AMPK-activated protein kinase (AMPK) ${ }^{8,9}$. We have previously demonstrated that heme induces ATF1 at both mRNA and protein levels, and causes its activation by Ser63 phosphorylation ${ }^{4}$. The coregulation of HO- 1 and LXR $\beta$ by phospho-ATF1 allows ATF1 to play a pivotal role in linking storage of iron with export of 
lipid. Since ATF1 directs these two key protective systems to work in concert, it holds enormous therapeutic promise ${ }^{10,11}$. We therefore asked which kinases phosphorylate (and thereby activate) ATF1 in response to heme.

\section{AMPK}

AMPK is a key mediator of cellular energy homeostasis, and responses to metabolic stress ${ }^{12}$, 13. When activated by low energy levels (low ATP / AMP ratio), it promotes ATP-creating catabolic pathways over ATP-expending anabolic pathways ${ }^{12,13}$. It is also activated by oxidative or genotoxic stress ${ }^{12}$. Structurally, AMPK is composed of three chains $(\alpha, \beta, \gamma)$, each with up to three isoforms ${ }^{14}$. AMPK may modify transcription via phosphorylation of a large and growing number of transcription factors and chromatin proteins ${ }^{13,15-21}$. Critically, these include $\mathrm{ATF} 1{ }^{9}$. Purified AMPK phosphorylated recombinant purified ATF1 in an in vitro kinase assay in a cell-free system, in a paper defining AMPK-mediated phosphorylation of CREB family proteins in vivo in skeletal muscle during exercise ${ }^{22}$. Moreover, AMPK activation in macrophages induces an anti-inflammatory response, with release of IL-10 ${ }^{23}$. Thus AMPK is a strong candidate to mediate ATF1 phosphorylation and transcription in response to heme following IPH and erythrophagocytosis.

\section{Metformin}

Metformin is effective in Type-II diabetes ${ }^{24,25}$, without causing hypoglycaemia. Indeed, metformin has proven sufficiently safe over some 50 years that it is now prescribed for debilitating but non-life threatening endocrine disease and metabolic syndrome ${ }^{26,27}$. In two independent recent large scale clinical trials, metformin has exhibited a significantly greater reduction of cardiovascular events (which are mainly due to atherosclerosis), than would be expected from the improved glycemic control alone ${ }^{28}$. This suggests that metformin may have a direct vascular protective effect, possibly located in the vessel wall. 
AMPK is also known to be activated by metformin ${ }^{26}$. However, although metformin clearly can activate AMPK, it is not clear how much of its clinical effect critically requires AMPK versus other pathways ${ }^{29,30}$. Interestingly, there is some evidence that AMPK may be antiinflammatory in classical T-lymphocyte / macrophage mediated inflammation models such as extrinsic allergic encephalomyelitis ${ }^{31}$. This raises the possibility that metformin may have wider potential as an anti-inflammatory agent in classical inflammatory diseases such as transplant rejection and autoimmunity, as well as in atherosclerotic inflammation ${ }^{31}$.

\section{Hypothesis and summary}

We tested the hypothesis that an AMPK-ATF1 pathway mediates heme-induced HO-1 and LXR $\beta$ and examined the implications for potential mechanisms of action of metformin. We show here that heme at a pathophysiologically relevant concentration $\left(\begin{array}{lll}10 & \mu \mathrm{M}\end{array}\right)^{4}$ and metformin at a pharmacologically relevant concentration (also $10 \mu \mathrm{M}$ ) ${ }^{37}$ increase ATF1 transcripts, ATF1 protein and activated (phospho-Ser63) ATF1, via AMPK, and that thereby metformin induces ATF1-dependent pathways including HO-1 and LXR $\beta$. Thus AMPK is a key initiator of ATF1 signaling evoked by IPH, and AMPK $\rightarrow$ ATF1 may be therapeutically activated by metformin. 


\section{Methods}

We cultured human blood-derived macrophages from normal human volunteers, with Ethical approval and informed consent; and monocyte purification was as we previously described ${ }^{3}$. We used established transfection methods for siRNA and plasmids in order to obtain molecular specificity, supplemented with pharmacological reagents. Mouse bone marrow derived macrophages (BMM) were cultured as widely established. Cell culture, RNA analysis, western blotting were carried out as before ${ }^{4}$. 


\section{Results}

\section{Heme activates AMPK in human blood-derived and plaque macrophages}

AMPK has a catalytic $\alpha$ subunit that can be activated by autophosphorylation, with $-\alpha 1$ being the main $\alpha$ chain in human macrophages. We probed lysates of human macrophages by immunoblotting with anti-phospho-AMPK- $\alpha 1$. We found that heme $(10 \mu \mathrm{M})$ increased (activated) phospho-AMPK, in a biphasic pattern, with an initial peak within minutes and a later one at $2-4 \mathrm{~h}$ coincident with the p-ATF1 (Figure 1A). Heme at this pathologically relevant concentration induced ATF1 protein progressively over 4h; phospho-ATF1 over 0.125-4h; and HO-1 target protein at $4 \mathrm{~h}$ (Figure 1A). Thus, the timing of AMPK activation by heme was sufficiently early to be consistent with an initiating event. We next assessed the in vivo relevance of AMPK by carrying out confocal microscopy of human plaques (Figure 1B). We found that phospho-AMPK was expressed by Mhem macrophages in areas of IPH, but not control foam cell areas, identified by comparison with serial sections that had been immunostained for p-ATF1, HO-1, LXR, ABCA-1, 8-oxo-G, CD163 and HLA-DR in previous publications $\left({ }^{3,4}\right.$ and not shown again). We have previously shown that $\mathrm{p}$-ATF1 is a key functional and immunohistochemical identifier of Mhem, since it directs the majority of heme-induced genes and is found selectively in Mhem cells in plaques ${ }^{4}$. The sections were therefore also double-stained for p-ATF1 and p-AMPK, which indeed showed colocalisation in the same cells (Figure 1B). As might be expected, p-AMPK was predominantly in the cytoplasm and p-ATF1 predominantly in the nucleus (Figure 1B).

\section{Pharmacological inhibition of AMPK inhibits induction of phospho-ATF1, ATF1, HO-1 and LXR $\beta$ by heme and metformin}

Since heme $(10 \mu \mathrm{M})$ activated AMPK and ATF1, we next determined whether there was a causal connection, initially using a simple pharmacological strategy and protein quantification by western analysis (representative blots in Figure 2A, densitometry in Figure 
2B). We found that metformin at $10 \mu \mathrm{M}$ (a known AMPK activator) induced phospho-ATF1, and its target genes HO-1 and LXR; and that these activation events in response to either heme $(10 \mu \mathrm{M})$ or metformin $(10 \mu \mathrm{M})$ were antagonised by $1 \mu \mathrm{M}$ compound-C (dorsomorphin), a known inhibitor of AMPK (Figure 2A, B).

We then assessed whether the induction of target proteins by metformin was via mRNA (i.e. transcriptional) (Supplemental Figure S-I). We found that metformin induced mRNA for ATF1 (Supplemental Figure S-IA,B), followed by that for HO-1 (Supplemental Figure S-IC). Heme $(10 \mu \mathrm{M})$ and metformin $(10 \mu \mathrm{M})$ induced mRNA for ATF1, HO-1 and LXR $\beta$, and induction of each was inhibited by the specific AMPK-inhibitor compound-C (dorsomorphin) at $1 \mu \mathrm{M}$ (Supplemental Figure S-IB,D,E). Thus heme and metformin induce ATF1, HO-1 and LXR $\beta$ via an AMPK-dependent transcriptional pathway.

\section{AMPK-knockdown inhibits induction of phospho-ATF1, HO-1 and LXR by heme and metformin}

To show that heme and metformin specifically activated ATF1 via AMPK, we next corroborated the pharmacological inhibition with an siRNA approach, using transfection protocols that we have validated for human blood-derived macrophages (Figure 3) ${ }^{32}$. Heme $(10 \mu \mathrm{M})$ induced phospho-ATF1, and this was suppressed by AMPK- $\alpha 1$ knock-down by siRNA, with oligos from Qiagen (Figure 3A,C). These data were extended and corroborated with siRNA oligos from an independent supplier (namely Dharmacon) individually targetting each subunit of AMPK that had been identified as expressed in macrophages by microarray ${ }^{4}$ (Supplemental Figure S-II). These experiments showed that the stimulants heme $(10 \mu \mathrm{M})$ (Supplemental Figure S-II) and metformin $(10 \mu \mathrm{M})$ (Supplemental Figure S-II) each induced phospho-ATF1, and in each case phospho-ATF1 induction was inhibited by AMPKknockdown, to variable degrees depending on which individual AMPK subunit was suppressed. We then examined the effects of suppressing each of the AMPK subunits, and of 
all of the AMPK subunits together, again using siRNA transfection in primary human macrophages (Supplemental Figure S-III). This combined AMPK-knockdown suppressed AMPK, which inhibited the capacity of heme $(10 \mu \mathrm{M})$ to increase phospho-ATF1, HO-1 and LXR $\beta$ proteins (Supplemental Figure S-III, quantified by densitometry in Supplemental Figure S-III).

We then carried out essentially the same procedure for metformin (Figure 3B,D). Metformin $(10 \mu \mathrm{M})$ induced each of p-ATF1, p-AMPK, HO-1 and LXR $\beta$, and the induction of each of these was suppressed by AMPK-knockdown (Figure 3B,D).

We then examined the AMPK-dependence of key heme-modulated macrophage functions that were potentially therapeutic ${ }^{4}$. We found that AMPK-knockdown caused heme $(10 \mu \mathrm{M})$ to become pro-oxidant (Figure 4A); prevented the cholesterol export induced by heme $(10 \mu \mathrm{M})$ (Figure 4B); and reversed suppression of cholesterol accumulation caused by heme $(10 \mu \mathrm{M})$ (Figure 4C); and prevented heme from inducing IL-10 secretion (Figure 4D). Whilst heme suppresses foam cell formation, heme in fact promoted uptake of di-O-labelled OxLDL indicating that it does not prevent phagocytic clearance of extracellular OxLDL (Figure 4E). AMPK-knockdown and ATF1-knockdown inhibited this effect (Figure 4E).

\section{Beta $(\beta)$-oxidation pathway of cellular lipid disposal is activated by heme via AMPK} and ATF1

Our microarray data on heme-stimulated macrophages identified induction of multiple $\beta$ oxidation genes, one of the principal pathways for fatty acid degradation ${ }^{4}$. AMPK classically activates $\beta$-oxidation pathways (fatty acid degradation) by a non-genomic mechanism involving phosphorylation of acetyl-CoA-carboxylase (ACC) ${ }^{33}$. While LXR directs cholesterol export, its ability to combat lipid overload is blunted by co-induction of the fatty

acid synthase (FASN) gene ${ }^{34,35}$. Thus, coordinated activation of both fatty acid oxidation 
(regulated by AMPK directly) and cholesterol export (regulated via LXR) is likely to be complementary for foam cell protection.

We therefore assessed the role of heme in $\beta$-oxidation by accepted methodology, using decay of a fluorescently-tagged fatty acid BODIPY-C11 as in Supplemental Figure S-IV. We found that heme $(10 \mu \mathrm{M})$ induces the degradation of this fluorescent fatty acid tracer in a timedependent manner, and this was blocked by etomoxir, a specific inhibitor of mitochondrial $\beta$ oxidation (Supplemental Figure S-IVA) ${ }^{36}$. This heme-induced $\beta$-oxidation was blocked by ATF1-siRNA (Supplemental Figure S-IVB) and by AMPK-knockdown by combined siRNA (Supplemental Figure S-IVC). This indicated that it is dependent on both AMPK and ATF1 consistent with an AMPK-ATF1 pathway. As expected for a known AMPK activator, metformin $(10 \mu \mathrm{M})$ also induced $\beta$-oxidation, which was also blocked by AMPK-knockdown by combined-siRNA (Supplemental Figure S-IV D).

\section{The AMPK activator metformin reproduces ATF1 signaling in Mhem}

In normal human volunteers, the peak plasma metformin concentration after oral absorption of a standard prescribed tablet $\left(\mathrm{CP}_{\max }\right)$ is $10 \mu \mathrm{M}^{37}$. Figure $5 \mathrm{~A}$ shows a concentration-effect curve of metformin on the release of highly reactive oxygen species (hROS) from macrophages derived from normal human volunteers, with the peak plasma concentration highlighted. In contrast to simple dihydro-dyes for redox potential, hROS specifically detect the most pathological species (peroxynitrite, hypochlorite and hydroxyl radicals) via electrophilic adduction of the amino group of the dye aminophenylfluorescein (APF) ${ }^{38}$. Notably, substantial antioxidant protection $(25-50 \%)$ was identified at the $\mathrm{CP}_{\max }(10 \mu \mathrm{M})$ and at considerably lower metformin concentrations than the $\mathrm{CP}_{\max }(100 \mathrm{nM}-1 \mu \mathrm{M})$.

The suppression of macrophage hROS by metformin at $\mathrm{CP}_{\max }(10 \mu \mathrm{M})$ was reversed by AMPK-knockdown by combined-siRNA (Figure 5B). We had previously found that heme 
$(10 \mu \mathrm{M})$ suppresses the macrophage antigen presentation molecule and activation marker HLA-DR via an ATF1 pathway. We therefore examined the effects of metformin on macrophage HLA-DR, finding that $10 \mu \mathrm{M}$ metformin substantially suppressed surface HLADR on macrophages (Figure 5C).

Next, since we had shown that $10 \mu \mathrm{M}$ metformin also induced LXR $\beta$ mRNA and protein, we assessed whether this had resultant beneficial effects on lipid metabolism. We found that metformin prevented foam cell formation due to incubation with oxidised LDL (OxLDL) in five separate macrophage cultures from normal volunteers (Figure 5D), as previously published with heme $(10 \mu \mathrm{M}){ }^{4}$. We next analysed the mechanism of this effect with the AMPK-knockdown by combined-siRNA protocols used in preceding experiments, quantifying foam cell formation as published (Figure 5E). Both $10 \mu \mathrm{M}$ heme and $10 \mu \mathrm{M}$ metformin suppressed OxLDL-induced foam cell formation; and both effects were prevented with AMPK-knockdown by combined-siRNA (Figure 5E). We then corroborated this effect by analysing cellular cholesterol, and showed that this effect of metformin was AMPKdependent since it was abolished by AMPK-knockdown by combined-siRNA (Figure 5F). We have previously validated an ApoA1-dependent export assay that measures the LXR / ABCA1 pathway ${ }^{4}$. Macrophage cholesterol export to ApoA1 in the culture medium was stimulated by metformin in macrophage from five normal human volunteers and prevented by AMPK-knockdown by combined-siRNA (Figure 5G). Thus metformin reproduces multiple potentially therapeutic features of the effect of heme on macrophages, and both do so in an AMPK-dependent and ATF1-dependent pathway. 


\section{Heme and metformin induce less p-ATF1, HO-1 and LXR in AMPK-KO compared to wild type murine bone marrow macrophages}

In human blood-derived macrophages, which are highly relevant, AMPK-knockdown reduced the ability of heme and metformin to activate p-ATF1, HO-1 and LXR $\beta$ and promote linked antioxidant and foam cell protective effects. However, AMPK was only knocked down and not out in these experiments, it was difficult to be definitively identify the contribution of AMPK-dependent and AMPK-independent pathways. We therefore assessed responses to heme and metformin in bone marrow macrophages (BMMs) from AMPK-knockout (AMPKKO) and matched wild-type (WT) mice (Figure 6).

In WT BMMs, heme $(10 \mu \mathrm{M})$ activated pThr172-AMPK, with subsequent induction of pSer63-ATF1, t-ATF1, HO-1 and LXR $\beta$ (n= 10 mice) (Figure 6A). This was less apparent in BMMs from AMPK-KO mice (Figure 6A). As controls, AMPK was absent in AMPK-KO mice, as was AMPK activation (Figure 6A). In WT BMMs, metformin $(100 \mu \mathrm{M})$ activated pT172-AMPK, with subsequent activation of pSer63-ATF1, t-ATF1, HO-1 and LXR $\beta$ ( $n=10$ mice) (Figure 6B). This was less apparent in BMMs from AMPK-KO mice (Figure 6B). Thus in murine BMMs, heme and metformin activate the same ATF1/HO-1/LXR cascade that they do in human, and the activation is dependent on AMPK.

The functional consequences of this activation were next tested (Figure 6C-F). In WT BMMs, incubation with heme $(10 \mu \mathrm{M})$ for $48 \mathrm{~h}$ suppressed oxidative stress as measured by APF, which detects highly reactive oxygen species (Figure 6C). In contrast, in BMMs from AMPK-KO mice, heme and metformin had the opposite effect, increasing hROS (Figure 6C). A similar mechanism was found with metformin (Figure 6D). In WT BMMs, incubation with metformin $(10 \mu \mathrm{M})$ for $48 \mathrm{~h}$ suppressed oxidative stress as measured by APF, which 
detects highly reactive oxygen species (Figure 6D). In contrast, in BMMs from AMPK-KO mice, heme and metformin had the opposite effect, increasing hROS (Figure 6D).

Next the effects of heme and metformin on survival of WT and AMPK-KO BMMs were assessed, using the XTT vital dye. This revealed an effect converse to that on oxidative stress, that is heme or metformin increased survival in WT cells, but reduced survival in AMPK-KO cells (Figure 6E).

We then studied the resistance of WT and AMPK-KO murine BMMs to lipid accumulation (foam cell formation) (Figure 6E). Oxidatively modified LDL evoked formation of foam cells (as with human macrophages) (Figure $6 \mathrm{~F})$. Heme $(10 \mu \mathrm{M})$ and metformin $(10 \mu \mathrm{M})$ also suppressed foam cell formation (Figure 6F) (as with human macrophages). Protection from foam cell formation by either heme or metformin was lost in AMPK-KO BMMs (akin to AMPK-knockdown in human macrophages) (Figure 6F).

Thus, we have previously shown that the transcription factor ATF1 is a key mediator of development of the Mhem phenotype in human atherosclerotic plaques ${ }^{6}$. We here show here that heme and metformin activate ATF1 via AMPK in both human and murine cells in vitro, with transcriptional induction of HO-1 and LXR that allows interlinking of antioxidant activity and resistance to foam cell formation. 


\section{Discussion}

\section{Summary}

We show here that ATF1, which we previously established is the key driver of Mhem macrophages, and is activated by heme $(10 \mu \mathrm{M})$ via AMPK-dependent phosphorylation. We have defined a clinical application of this pathway, in which metformin, a common safe oral hypoglycemic agent, may coregulate anti-inflammatory, antioxidant and foam-cell protective mechanisms in macrophages. The data is therefore highly significant for clinical treatment of atherosclerotic risk, and potentially inflammation in general.

\section{AMPK and metabolic stress}

While AMPK is primarily a responder to low fuel ${ }^{39-41}$, it is also a wider stress-activated kinase, being also activated by oxidative stress, osmotic stress ${ }^{42}$, ionizing radiation ${ }^{43}$ and hypoxia ${ }^{44}$, and may therefore play a role in signalling akin to classical stress-activated protein kinases such as p38MAPK and JNK. We suggest that the metabolic stress of erythophagocytosis parallels that of energy deprivation, since both are associated with oxidative stress and require activation of catabolic pathways. Indeed, the term 'heme stress' may be appropriate.

\section{AMPK and macrophage phenotype.}

It was previously shown that transfecting a mouse macrophage cell line with expression plasmids for constitutively active AMPK modified macrophage signalling, increasing phospho-CREB, increasing phospho-PI3K and suppressing phospho-I $\mathrm{B} \alpha$; together with induction of IL-10 and suppression of TNF- $\alpha^{23}$. The converse was obtained with dominant negative AMPK ${ }^{23}$. Moreoever, a classical anti-inflammatory stimulus promoted AMPK phosphorylation and induced AMPK, whereas LPS (a classical inflammatory stimulus) had 
the reverse effect ${ }^{23}$. We now extend these data and translate them to metformin pharmacology.

\section{ATF1 and stress kinases other than AMPK}

ATF1 is phosphorylated by several stress kinases including AMPK in skeletal muscle during exercise $^{22}$, p38MAPK in leptin signalling ${ }^{45}$, mitogen stimulated kinase (MSK) in UVinduced stress ${ }^{46,47}$, and by protein kinase $\mathrm{D}^{48}$. Tyrosine phosphorylation of ATF1 by HIPK2 has also been described in response to genotoxic stress ${ }^{49}$. Understanding the full role of AMPK / ATF1 in the context of other upstream kinases regulation in the differentiation of Mhem is a major priority for further work. A combinatorial role of alternative kinases seems possible, since there were subtle mismatches between increases in phospho-ATF1 and in the target genes.

\section{AMPK and TFs other than ATF1}

Although AMPK was first, and best, characterised as a post-translational regulator of metabolic enzymes, recent work reveals that it has important and numerous transcriptional effects (ie mediated by phosphorylation of transcription factors) ${ }^{14}, 18$. Indeed, there is a described AICAR-response element and corresponding AICAR-response element binding protein, which is a zinc-finger protein ${ }^{50}$. AMPK mediates in vitro and in vivo phosphorylation of CREB-family proteins in skeletal muscle in exercise, and in the same paper was shown to direct phosphorylation of purified ATF1 by purified AMPK in a cell-free

system $^{22}$. The functional consequences are potentially profound since microarray-validated target genes for AMPK, modulated in skeletal muscle in AMPK-KO mice in vivo include: PEPCK (the rate limiting enzyme for gluconeogenesis), fatty acid synthase (the rate limiting enzyme for fatty acyl / triglyceride production), eNOS (the endothelial cytoprotective vasodilator), ferritin (which safely stores iron), d-amino-levulinic acid synthase (d-ALA- 
synthetase, ALAS, the enzyme for the rate limiting step of heme biosynthesis) ${ }^{51}$. Thus, AMPK regulate genes controlling vascular tone, iron storage, porphyrin / heme biosynthesis in addition to its traditional roles in glucose metabolism ${ }^{51}$. Moreover, AMPK may inactivate $\mathrm{NF}_{\kappa} \mathrm{B}^{52}$; and suppresses oxidative stress via upregulation of thioredoxin ${ }^{53}$. Given the antiinflammatory effects of AMPK, and its direction of a plethora of protective transcriptional effects, it is plausible that AMPK might initiate commitment to Mhem. Of interest, we found by systems biology approaches that the heme-induced geneset was enriched in ChIP-Seq validated binding sites for approximately 7 TFs, which in turn contained conserved AMPKphosphorylation motifs (not shown).

\section{Metformin and AMPK-dependence}

The mechanism of action of metformin is still in the process of elucidation ${ }^{26}$. Some data, using the small molecule AMPK inhibitor dorsomorphin, indicated that the hypoglycemic effects of metformin may be largely AMPK-mediated ${ }^{54}$. Metformin mediated activation was also found to be dependent on the tumour-suppressor gene liver kinase B (LKB), which is often taken as an exclusive upstream kinase of AMPK ${ }^{55}$. More recently, doubt was thrown on the specificity of metformin for AMPK since many of its hepatic hypoglycemic effects are identical in AMPK-knockout mice ${ }^{30}$. In this paper, we carefully showed AMPK-dependence of the effects of metformin in human primary macrophages in vitro, using corroborating siRNA transfection and pharmacological strategies.

\section{Possible clinical applications}

Metformin is relatively safe oral hypoglycaemic agent that, unlike sulphonylureas, does not provoke hypoglycemia, even in normoglycemic subjects or in overdose ${ }^{27}$. This is thought to be related to its activation of cellular energy homeostasis ${ }^{27}$. In two recent large-scale clinical trials of cardiovascular endpoints in diabetes, metformin had a greater reduction in 
cardiovascular events than was predicted from its effects on glycemic control alone ${ }^{28}$. This suggested the possibility of a putative direct effect on vessels ${ }^{56}$. Other studies have suggested variable but favourable effects on lipid profile, with reduced LDL in diabetes ${ }^{28,57}$ or enhanced HDL in polycystic ovarian syndrome ${ }^{28,58}$. These findings provide a potential mechanism for these clinical effects. Namely, metformin may direct a major pathogenic cell type in atherosclerotic lesions to initiate joint anti-oxidant, anti-inflammatory and cytoprotection, with promotion of cholesterol efflux to HDL. Metformin may therefore be an agent that could suppress atherosclerotic plaque inflammation useful in a wider range of risk factors than in solely overt diabetes. Moreover, since there is evidence that metformin is also cardioprotective by improving myocardial remodelling and myocardial protection in ischemia-reperfusion $^{28}$, this may reflect commitment to Mhem macrophages in damaged myocardium. Our work is yet limited by being restricted to in vitro macrophage - albeit human monocyte derived macrophages from multiple normal volunteers. Taking the work further will require progression to in vivo model such as $\mathrm{LDR}^{-/-}, \mathrm{ATF}^{-/-}$and $\mathrm{AMPK}^{-/}$mice. Moreover, pigs readily develop vascular and cardiometabolic disease in response to diet like humans - and would make a representative model. Finally, analysing peripheral blood monocytes in patients before and after metformin commencement would yield insights into the clinical relevance. In vivo and transcriptomic studies are now urgently required to define potential therapeutic implications and these are planned.

\section{Conclusions}

We here show here that heme and metformin activate ATF1 via AMPK in both human and murine cells in vitro. The AMPK-ATF1 pathway drives transcriptional co-induction of HO-1 and LXR that allows interlinking of antioxidant activity, resistance to foam cell formation and macrophage deactivation. We have previously identified that ATF1 initiates a coordinated transcriptional response and macrophage phenotype that we termed Mhem. Since 
metformin is an important antidiabetic drug, these data yield a new mechanistic insight into recent clinical trial data indicating an enhanced suppression of diabetic macrovascular disease by metformin. Whether this pathway protects against vascular disease in vivo is under active investigation.

\section{Acknowledgements}

None

\section{Sources of funding}

We thank the British Heart Foundation for financial support (Gerry Turner Intermediate Fellowship FS07/010 to JJB, BHF Senior Clinical Research Fellowship SCRF03 to JJB) and Professorial Core Support to DOH) and thank the Cambridge Biomedical Research Centre and Imperial College NIHR Biomedical Research Centre for support.

\section{Disclosures}

None 


\section{Figure Legends}

\section{Figure 1 Human Mhem have activated AMPK in vitro and in vivo}

(A) Early time course of human macrophage response to heme Human monocyte derived macrophages were purified from peripheral blood and cultured for $24 \mathrm{~h}$. Then they were stimulated with heme or vehicle control and protein lysates were made at the indicated time points $(0,0.125,0.25,0.4,1,2$ and $4 \mathrm{~h})$. Lysates were run on SDSPAGE, electrotransferred to PVDF membranes which were then immunoblotted with the indicated antibodies. Bands are at the appropriate $\mathrm{Mr}$ (indicated).

(B) Mhem macrophages in coronary plaques with intraplaque hemorrhage contain increased activated AMPK relative to plaque foam cells Confocal micrographs: upper image pair shows anti-phospho-AMPK (activated AMPK) immunofluorescence (Alexa568, red) or DNA-binding dye (TOPRO, blue) as indicated. Scalebars as indicated. Cells were in the matching areas of serial sections to

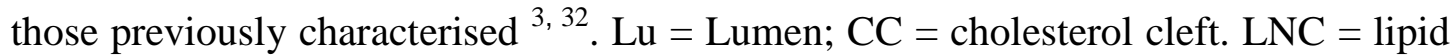
necrotic core. Mhem and FC areas of the same plaque were imaged at the same settings and were identified from p-ATF1, CD163, HLA-DR, HO-1, LXR $\beta$, ABCA1, ApoE, 8-oxo-G immunostaining of serial sections. Lower image pair: dual immunostaining with anti-phospho-AMPK (activated AMPK, green) and antiphospho-ATF1 (red). 
Figure 2 Heme and metformin induce, and pharmacological AMPK inhibition suppresses, phospho-ATF1, HO-1 and LXR $\beta$ in human macrophages

Human monocyte derived macrophages were purified from peripheral blood and rested for 24h. Then they were cultured with heme $(10 \mu \mathrm{M})$, metformin $(10 \mu \mathrm{M})$, the AMPK-inhibitor compound-C (Dorsomorphin $1 \mu \mathrm{M}$ ) and protein lysates made at 4 h. Lysates were run on SDSPAGE, electrotransferred to PVDF membranes, which were immunoblotted with antibodies to phospho-ATF1, total ATF1, phospho-ACC, HO-1 and LXR $\beta$ as indicated (Methods). Representative blots ( $\mathrm{n}=5$ donors) showing bands at the appropriate $\mathrm{Mr}$ (indicated) are shown in (A). (B) Densitometry for the bands as before; expressed as mean \pm SE for $n=5$ donors; $*<\mathrm{p}<0.05$, paired Wilcoxon test, $\mathrm{n}=5$ donors.

Figure 3 Heme and metformin induce AMPK activation and induce phospho-ATF1, ATF1, HO-1 and LXR $\beta$ dependent on AMPK

Human blood-derived macrophages were transfected with matched control-siRNA or Qiagen AMPK- $\alpha 1$ siRNA oligos $0.1 \mathrm{nmol} / 10^{5}$ cells for $24 \mathrm{~h}$ as indicated; and then cells were stimulated with heme $10 \mu \mathrm{M}$ for $4 \mathrm{~h}$ as indicated and stimulated with either heme (A, C) or metformin (B, D), then lysed at $4 \mathrm{~h}$ and western blotted for the proteins and phospho-proteins indicated. A, B, represent $n=6$ experiments each. C, D, densitometry, mean \pm SE, $n=6$ experiments. $*<\mathrm{p}<0.05$, paired Wilcoxon test, $\mathrm{n}=5$ donors. 
Figure 4 Heme induces potentially therapeutic macrophage functional responses dependent on AMPK

(A) AMPK-knockdown renders heme pro-oxidant. Human blood derived macrophages were cultured with a combined-AMPK-siRNA protocol (Methods). Then heme $(10 \mu \mathrm{M})$ was added for a further $24 \mathrm{~h}$ and highly reactive oxygen species (peroxynitrite, hypochlorite, hydroxyl radicals) were measured using APF dye as before ${ }^{6} .{ }^{*} p<0.05$, Student's t-test, AMPK-knockdown by combined-siRNA reverses heme-mediated hROS suppression [note in the absence of doublestranded RNA, heme is normally outright anti-oxidant).

(B) AMPK-dependence of induction of cholesterol export by heme. Human bloodderived macrophages were cultured with a combined-AMPK-siRNA protocol (Methods). Then heme $(10 \mu \mathrm{M})$ was added for a further $24 \mathrm{~h}$ and the medium was changed and then cholesterol export to supernatant containing lipoprotein-free fetal calf serum and $10 \mu \mathrm{g} \cdot \mathrm{mL}^{-1}$ ApoA1 (both Sigma-Aldrich), using an ultrasensitive enzymatic assay based on cholesterol oxidase and Amplex Red as before ${ }^{6}$. $*_{p}<0.05$, Student's t-test, AMPK-siRNA reverses heme-induced cholesterol export, $\mathrm{n}=5$ donors.

(C) AMPK-dependence suppression of cholesterol accumulation by heme. Human blood-derived macrophages were cultured with a combined-AMPK-siRNA protocol (Methods). Then heme $(10 \mu \mathrm{M})$ was added to the culture medium, along with $30 \mu \mathrm{g} \cdot \mathrm{mL}^{-1}$ OxLDL. Supernatant and cell-associated cholesterol were measured with an ultrasensitive enzymatic assay based on cholesterol oxidase and Amplex Red as before ${ }^{6} .{ }^{*} \mathrm{p}<0.05$, Student's t-test, heme suppresses cholesterol accumulation, strongly reversed by AMPK-knockdown by combined-siRNA, $n=5$ donors. In macrophages with AMPK-knockdown, heme causes an increase in cell cholesterol. 
(D) IL-10 secretion in response to heme is AMPK-dependent. Human bloodderived macrophages were cultured with the combined-AMPK-siRNA protocol (Methods). Macrophages were cultured as before in 96-well plates, supernatants were collected and assayed for IL-10 using a DuoSet (R\&D Systems). Data are mean \pm SE, $\mathrm{n}=5$ donors. ${ }^{*}<\mathrm{p}<0.05$, Student's t-test.

(E) Heme increases OxLDL uptake in an AMPK-dependent and ATF1dependent manner. Human blood-derived macrophages were cultured with the combined-AMPK-siRNA protocol (Methods). Data are representative flow cytometric histograms $n=3$ donors. Treatments are as indicated. 


\section{Figure 5 Modulation of macrophage functions by metformin}

Human blood derived macrophages were cultured in the presence or absence of metformin and key functions relevant to atherosclerosis were measured, in the presence or absence of combined knockdown of all the AMPK chains where indicated AMPK- $\alpha \beta \gamma$. Human bloodderived macrophages were transfected with pooled control-siRNA or Dharmacon combined siRNA oligos targetting all major AMPK chains (AMPK- $\alpha \beta \gamma$ ) at $0.01 \mathrm{nmol} / 10^{4}$ cells for $24 \mathrm{~h}$, as indicated.

(A) Metformin suppresses oxidative burst in human blood-derived macrophages at clinically relevant concentrations. Human blood-derived macrophages were cultured with increasing concentrations of metformin and oxidative stress was measured as highly reactive oxygen species after $48 \mathrm{~h}$ (peroxynitrite, hypochlorite, hydroxyl radicals) with aminophenylfluorescein $(\mathrm{APF})$ as before ${ }^{6}$. Red box $=$ peak plasma concentration after oral dose $(10 \mu \mathrm{M})$. Data are mean \pm SE. $*<\mathrm{p}<0.05$, Student's t-test, $\mathrm{n}=5$ independent experiments.

(B) AMPK-dependence of suppression of oxidative stress by metformin. Human blood-derived macrophages were transfected with control-siRNA oligos or combined-AMPK-siRNA oligos as indicated; using the protocol validated in Figure 3. Then the cells were cultured in the presence or absence of metformin for a further 24h and oxidative stress measured using APF as in Figure 6A. Data are mean \pm SE. ${ }^{*}<\mathrm{p}<0.05$, Student's t-test, $\mathrm{n}=5$ independent experiments.

(C) Metformin suppresses activation marker HLA-DR in human blood-derived macrophages at clinically relevant concentrations. Human blood-derived macrophages were cultured in the presence or absence of $10 \mu \mathrm{M}$ metformin as indicated; macrophages were harvested after 4 days of culture, and fluorescently 
stained for HLA-DR and analysed by flow cytometry. Representative of $n=3$ donors.

(D) Metformin suppresses foam cell formation in human blood-derived macrophages at clinically relevant concentrations. Human blood-derived macrophages were cultured in the presence or absence of $10 \mu \mathrm{M}$ metformin (bottom panels) and $30 \mu \mathrm{g} \cdot \mathrm{mL}^{-1}$ OxLDL (right hand panels) for 1 week and then fluorescently stained for lipid with Nile Red and counterstained with a nuclear counterstain. Metformin prevents OxLDL-induced foam cell formation. Image representative of $n=5$ donors. [green $=$ completely neutral lipid eg triglyceride, yellow $=$ mildly polar lipids eg phospholipid, red $=$ TOPRO nuclear dye $]$.

(E) Metformin suppresses foam cell formation in human blood-derived macrophages via AMPK. Human blood-derived macrophages ( $\mathrm{n}=5$ donors) were cultured in the presence or absence of $10 \mu \mathrm{M}$ metformin, $10 \mu \mathrm{M}$ heme, and $30 \mu \mathrm{g} \cdot \mathrm{mL}^{-1}$ OxLDL for 1 week, as in (D), and also in the presence of AMPKknockdown by combined AMPK- $\alpha \beta \gamma$ siRNA or matched pooled control-siRNA as indicated. Cells were fluorescently stained for lipid as in (D) and analysed by counting foam cells with a confocal microscope as before ${ }^{6}{ }^{*} \mathrm{p}<0.05$, ANOVA, metformin and heme suppress OxLDL-induced foam cell formation prevented by AMPK-knockdown by combined-siRNA. Note, heme had no significant effect on foam cell formation in the absence of OxLDL feeding, a control that is already published and omitted in the interests of space and duplication ${ }^{6}$. Data are mean \pm SE, $\mathrm{n}=9$ donors. $*<\mathrm{p}<0.05$, Student's t-test.

(F) Metformin increases cholesterol export to HDL via AMPK. Human bloodderived macrophages were cultured in the presence or absence of $10 \mu \mathrm{M}$ metformin, the medium was changed and then cholesterol export to supernatant 
containing lipoprotein-free fetal calf serum and $10 \mu \mathrm{g} \cdot \mathrm{mL}^{-1}$ ApoA1 (both SigmaAldrich). Cholesterol in the cells and supernatant were compared using an ultrasensitive enzymatic assay based on cholesterol oxidase and Amplex Red as before ${ }^{6}$. ${ }^{*} \mathrm{p}<0.05$, metformin induces total cell cholesterol export to ApoA1 (forming HDL), prevented AMPK-knockdown by combined-siRNA.

(G) Metformin decreases human blood-derived macrophage cholesterol content via AMPK. Human blood-derived macrophages were cultured in the presence or absence of $10 \mu \mathrm{M}$ metformin and $30 \mu \mathrm{g} \cdot \mathrm{mL}^{-1}$ OxLDL for 1 week, in the presence of AMPK-knockdown by AMPK- $\alpha \beta \gamma$ siRNA or matched control-siRNA as indicated. Then cholesterol was assessed in lysates using an ultrasensitive enzymatic assay based on cholesterol oxidase and Amplex Red as before ${ }^{4}$. Metformin reduces total cell cholesterol accumulation, prevented by AMPKknockdown by combined-siRNA. Data are mean \pm SE. $*<p<0.05$, Student's t-test. 
Figure 6 Heme and metformin induce p-ATF1, HO-1 and LXR- $\beta$ less in AMPK-KO than in WT mice

$(\mathrm{A}, \mathrm{B})$ are representative western blots of bone marrow macrophages (BMMs) from WT or AMPK-KO mice as indicated. BMMs were treated with heme $(10 \mu \mathrm{M})(\mathrm{A})$ or metformin $(100 \mu \mathrm{M})(\mathrm{B})$ over the indicated time course. Bands are at appropriate $\mathrm{Mr}$ (as stated). Representative of $\mathrm{n}=6 \mathrm{WT}$ and $\mathrm{n}=6 \mathrm{KO}$ mice. $(\mathrm{C}, \mathrm{D})$ generation of $\mathrm{hROS}(\mathrm{OCl}, \mathrm{OH}$, HONOO) by BMMs from WT or AMPK-KO mice as indicated, measured with APF dye, expressed in arbitrary fluorescence units (Methods). Y-axes, APF-related fluorescence, Xaxis, gene status. (C) hROS in BMM treated in presence or absence of heme $(10 \mu \mathrm{M})$; ${ }^{*} \mathrm{p}<0.05$, Student's t-test, Data are mean $\pm \mathrm{SE}, \mathrm{n}=5$ mice. (D) hROS in BMM from WT or AMPK-KO mice, treated in presence or absence of metformin $(10 \mu \mathrm{M}) ;{ }^{*} \mathrm{p}<0.05$, Student's ttest, Data are mean $\pm \mathrm{SE}, \mathrm{n}=5$ mice. (E) Y-axis, cell survival measured with XTT dye, expressed in arbitrary fluorescence units. X-axis, genotype. Treatment with heme or metformin are as indicated on Figure. Data are mean $\pm \mathrm{SE}, \mathrm{n}=5$ mice. ${ }^{*} \mathrm{p}<0.05$, Student's ttest. (F) Resistance to foam cell formation (lipid accumulation) in macrophages from WT or AMPK-KO mice. Y-axis, foam cell formation measured as before. $\mathrm{X}$-axis, treatments of cultures as indicated. Open and filled bars, knockout status as indicated. ${ }^{*} \mathrm{p}<0.05$, Student's ttest, Data are mean SE, $\mathrm{n}=5$ mice. 


\section{Reference List}

(1) Kolodgie FD, Gold HK, Burke AP, Fowler DR, Kruth HS, Weber DK, Farb A, Guerrero LJ, Hayase M, Kutys R, Narula J, Finn AV, Virmani R. Intraplaque hemorrhage and progression of coronary atheroma. N Engl J Med 2003;349:2316-25.

(2) Falk E, Nakano M, Bentzon JF, Finn AV, Virmani R. Update on acute coronary syndromes: the pathologists' view. Eur Heart J 2013;34:719-28.

(3) Boyle JJ, Harrington HA, Piper E, Elderfield K, Stark J, Landis RC, Haskard DO. Coronary intraplaque hemorrhage evokes a novel atheroprotective macrophage phenotype. Am J Pathol 2009;174:1097-108.

(4) Boyle JJ, Johns M, Kampfer T, Nguyen AT, Game L, Schaer DJ, Mason JC, Haskard DO. Activating transcription factor 1 directs Mhem atheroprotective macrophages through coordinated iron handling and foam cell protection. Circ Res 2012;110:20-33.

(5) Finn AV, Nakano M, Polavarapu R, Karmali V, Saeed O, Zhao X, Yazdani S, Otsuka F, Davis T, Habib A, Narula J, Kolodgie FD, Virmani R. Hemoglobin directs macrophage differentiation and prevents foam cell formation in human atherosclerotic plaques. J Am Coll Cardiol 2012;59:166-77.

(6) Boyle JJ, Johns M, Kampfer T, Nguyen AT, Game L, Schaer DJ, Mason JC, Haskard DO. Activating transcription factor 1 directs Mhem atheroprotective macrophages through coordinated iron handling and foam cell protection. Circ Res 2012;110:20-33.

(7) Rehfuss RP, Walton KM, Loriaux MM, Goodman RH. The cAMP-regulated enhancer-binding protein ATF-1 activates transcription in response to cAMPdependent protein kinase A. J Biol Chem 1991;266:18431-4.

(8) Rosenberg D, Groussin L, Jullian E, Perlemoine K, Bertagna X, Bertherat J. Role of the PKA-regulated transcription factor CREB in development and tumorigenesis of endocrine tissues. Ann N Y Acad Sci 2002;968:65-74.

(9) Thomson DM, Herway ST, Fillmore N, Kim H, Brown JD, Barrow JR, Winder WW. AMP-activated protein kinase phosphorylates transcription factors of the CREB family. J Appl Physiol 2008;104:429-38.

(10) Otterbein LE, Soares MP, Yamashita K, Bach FH. Heme oxygenase-1: unleashing the protective properties of heme. Trends Immunol 2003;24:449-55. 
(11) Calkin AC, Tontonoz P. Liver $\mathrm{x}$ receptor signaling pathways and atherosclerosis. Arterioscler Thromb Vasc Biol 2010;30:1513-8.

(12) Zhang BB, Zhou G, Li C. AMPK: an emerging drug target for diabetes and the metabolic syndrome. Cell Metab 2009;9:407-16.

(13) Bungard D, Fuerth BJ, Zeng PY, Faubert B, Maas NL, Viollet B, Carling D, Thompson CB, Jones RG, Berger SL. Signaling kinase AMPK activates stresspromoted transcription via histone H2B phosphorylation. Science 2010;329:1201-5.

(14) Kahn BB, Alquier T, Carling D, Hardie DG. AMP-activated protein kinase: ancient energy gauge provides clues to modern understanding of metabolism. Cell Metab 2005;1:15-25.

(15) Greer EL, Oskoui PR, Banko MR, Maniar JM, Gygi MP, Gygi SP, Brunet A. The energy sensor AMP-activated protein kinase directly regulates the mammalian FOXO3 transcription factor. J Biol Chem 2007;282:30107-19.

(16) Lin YY, Kiihl S, Suhail Y, Liu SY, Chou YH, Kuang Z, Lu JY, Khor CN, Lin CL, Bader JS, Irizarry R, Boeke JD. Functional dissection of lysine deacetylases reveals that HDAC1 and p300 regulate AMPK. Nature 2012;482:251-5.

(17) Canto C, Auwerx J. PGC-1alpha, SIRT1 and AMPK, an energy sensing network that controls energy expenditure. Curr Opin Lipidol 2009;20:98-105.

(18) Carling D, Zammit VA, Hardie DG. A common bicyclic protein kinase cascade inactivates the regulatory enzymes of fatty acid and cholesterol biosynthesis. FEBS Lett 1987;223:217-22.

(19) Ben S, I, Regazzetti C, Robert G, Laurent K, Le Marchand-Brustel Y, Auberger P, Tanti JF, Giorgetti-Peraldi S, Bost F. Metformin, independent of AMPK, induces mTOR inhibition and cell-cycle arrest through REDD1. Cancer Res 2011;71:4366-72.

(20) Hong YH, Varanasi US, Yang W, Leff T. AMP-activated protein kinase regulates HNF4alpha transcriptional activity by inhibiting dimer formation and decreasing protein stability. J Biol Chem 2003;278:27495-501.

(21) Thoreen CC, Sabatini DM. AMPK and p53 help cells through lean times. Cell Metab 2005;1:287-8.

(22) Thomson DM, Herway ST, Fillmore N, Kim H, Brown JD, Barrow JR, Winder WW. AMP-activated protein kinase phosphorylates transcription factors of the CREB family. J Appl Physiol 2008;104:429-38.

(23) Sag D, Carling D, Stout RD, Suttles J. Adenosine 5'-monophosphate-activated protein kinase promotes macrophage polarization to an anti-inflammatory functional phenotype. J Immunol 2008;181:8633-41.

(24) Fryer LG, Parbu-Patel A, Carling D. The Anti-diabetic drugs rosiglitazone and metformin stimulate AMP-activated protein kinase through distinct signaling pathways. J Biol Chem 2002;277:25226-32. 
(25) Shaw RJ, Lamia KA, Vasquez D, Koo SH, Bardeesy N, DePinho RA, Montminy M, Cantley LC. The kinase LKB1 mediates glucose homeostasis in liver and therapeutic effects of metformin. $S$ 2005;310:1642-6.

(26) Witters LA. The blooming of the French lilac. J Clin Invest 2001;108:1105-7.

(27) Viollet B, Guigas B, Sanz GN, Leclerc J, Foretz M, Andreelli F. Cellular and molecular mechanisms of metformin: an overview. Clin Sci (Lond) 2012;122:253-70.

(28) El MS, Rongen GA, de Boer RA, Riksen NP. The cardioprotective effects of metformin. Curr Opin Lipidol 2011;22:445-53.

(29) Miller RA, Birnbaum MJ. An energetic tale of AMPK-independent effects of metformin. J Clin Invest 2010;120:2267-70.

(30) Foretz M, Hebrard S, Leclerc J, Zarrinpashneh E, Soty M, Mithieux G, Sakamoto K, Andreelli F, Viollet B. Metformin inhibits hepatic gluconeogenesis in mice independently of the LKB1/AMPK pathway via a decrease in hepatic energy state. $J$ Clin Invest 2010;120:2355-69.

(31) Nath N, Khan M, Rattan R, Mangalam A, Makkar RS, de MC, Bertrand L, Singh I, Chen Y, Viollet B, Giri S. Loss of AMPK exacerbates experimental autoimmune encephalomyelitis disease severity. Biochem Biophys Res Commun 2009;386:16-20.

(32) Boyle JJ, Johns M, Kampfer T, Nguyen AT, Game L, Schaer DJ, Mason JC, Haskard DO. Activating Transcription Factor 1 directs Mhem atheroprotective macrophages via coordinated iron handling and foam cell protection. Circ Res 2012; In Press.

(33) Hardie DG. Minireview: the AMP-activated protein kinase cascade: the key sensor of cellular energy status. Endocrinology 2003;144:5179-83.

(34) Calkin AC, Tontonoz P. Transcriptional integration of metabolism by the nuclear sterol-activated receptors LXR and FXR. Nat Rev Mol Cell Biol 2012;13:213-24.

(35) Jakobsson T, Treuter E, Gustafsson JA, Steffensen KR. Liver X receptor biology and pharmacology: new pathways, challenges and opportunities. Trends Pharmacol Sci 2012.

(36) Samudio I, Harmancey R, Fiegl M, Kantarjian H, Konopleva M, Korchin B, Kaluarachchi K, Bornmann W, Duvvuri S, Taegtmeyer H, Andreeff M. Pharmacologic inhibition of fatty acid oxidation sensitizes human leukemia cells to apoptosis induction. J Clin Invest 2010;120:142-56.

(37) Graham GG, Punt J, Arora M, Day RO, Doogue MP, Duong JK, Furlong TJ, Greenfield JR, Greenup LC, Kirkpatrick CM, Ray JE, Timmins P, Williams KM. Clinical pharmacokinetics of metformin. Clin Pharmacokinet 2011;50:81-98.

(38) Setsukinai K, Urano Y, Kakinuma K, Majima HJ, Nagano T. Development of novel fluorescence probes that can reliably detect reactive oxygen species and distinguish specific species. J Biol Chem 2003;278:3170-5. 
(39) Hardie DG, Carling D, Gamblin SJ. AMP-activated protein kinase: also regulated by ADP? Trends Biochem Sci 2011;36:470-7.

(40) Carling D, Mayer FV, Sanders MJ, Gamblin SJ. AMP-activated protein kinase: nature's energy sensor. Nat Chem Biol 2011;7:512-8.

(41) Xiao B, Sanders MJ, Underwood E, Heath R, Mayer FV, Carmena D, Jing C, Walker PA, Eccleston JF, Haire LF, Saiu P, Howell SA, Aasland R, Martin SR, Carling D, Gamblin SJ. Structure of mammalian AMPK and its regulation by ADP. Nature 2011;472:230-3.

(42) Barnes K, Ingram JC, Porras OH, Barros LF, Hudson ER, Fryer LG, Foufelle F, Carling D, Hardie DG, Baldwin SA. Activation of GLUT1 by metabolic and osmotic stress: potential involvement of AMP-activated protein kinase (AMPK). J Cell Sci 2002;115:2433-42.

(43) Zannella VE, Cojocari D, Hilgendorf S, Vellanki RN, Chung S, Wouters BG, Koritzinsky M. AMPK regulates metabolism and survival in response to ionizing radiation. Radiother Oncol 2011;99:293-9.

(44) Emerling BM, Weinberg F, Snyder C, Burgess Z, Mutlu GM, Viollet B, Budinger GR, Chandel NS. Hypoxic activation of AMPK is dependent on mitochondrial ROS but independent of an increase in AMP/ATP ratio. Free Radic Biol Med 2009;46:1386-91.

(45) Cui H, Cai F, Belsham DD. Leptin signaling in neurotensin neurons involves STAT, MAP kinases ERK1/2, and p38 through c-Fos and ATF1. FASEB J 2006;20:2654-6.

(46) Staples CJ, Owens DM, Maier JV, Cato AC, Keyse SM. Cross-talk between the p38alpha and JNK MAPK pathways mediated by MAP kinase phosphatase-1 determines cellular sensitivity to UV radiation. J Biol Chem 2010;285:25928-40.

(47) Wiggin GR, Soloaga A, Foster JM, Murray-Tait V, Cohen P, Arthur JS. MSK1 and MSK2 are required for the mitogen- and stress-induced phosphorylation of CREB and ATF1 in fibroblasts. Mol Cell Biol 2002;22:2871-81.

(48) Johannessen M, Delghandi MP, Rykx A, Dragset M, Vandenheede JR, Van LJ, Moens U. Protein kinase D induces transcription through direct phosphorylation of the cAMP-response element-binding protein. J Biol Chem 2007;282:14777-87.

(49) Hailemariam K, Iwasaki K, Huang BW, Sakamoto K, Tsuji Y. Transcriptional regulation of ferritin and antioxidant genes by HIPK2 under genotoxic stress. $J$ Cell Sci 2010;123:3863-71.

(50) Shirai T, Inoue E, Ishimi Y, Yamauchi J. AICAR response element binding protein (AREBP), a key modulator of hepatic glucose production regulated by AMPK in vivo. Biochem Biophys Res Commun 2011;414:287-91.

(51) Nilsson EC, Long YC, Martinsson S, Glund S, Garcia-Roves P, Svensson LT, Andersson L, Zierath JR, Mahlapuu M. Opposite transcriptional regulation in skeletal muscle of AMP-activated protein kinase gamma3 R225Q transgenic versus knock-out mice. J Biol Chem 2006;281:7244-52. 
(52) Salminen A, Hyttinen JM, Kaarniranta K. AMP-activated protein kinase inhibits NFkappaB signaling and inflammation: impact on healthspan and lifespan. $J$ Mol Med (Berl) 2011;89:667-76.

(53) Hou X, Song J, Li XN, Zhang L, Wang X, Chen L, Shen YH. Metformin reduces intracellular reactive oxygen species levels by upregulating expression of the antioxidant thioredoxin via the AMPK-FOXO3 pathway. Biochem Biophys Res Commun 2010;396:199-205.

(54) Zhou G, Myers R, Li Y, Chen Y, Shen X, Fenyk-Melody J, Wu M, Ventre J, Doebber T, Fujii N, Musi N, Hirshman MF, Goodyear LJ, Moller DE. Role of AMP-activated protein kinase in mechanism of metformin action. J Clin Invest 2001;108:1167-74.

(55) Woods A, Johnstone SR, Dickerson K, Leiper FC, Fryer LG, Neumann D, Schlattner U, Wallimann T, Carlson M, Carling D. LKB1 is the upstream kinase in the AMPactivated protein kinase cascade. Curr Biol 2003;13:2004-8.

(56) Effect of intensive blood-glucose control with metformin on complications in overweight patients with type 2 diabetes (UKPDS 34). UK Prospective Diabetes Study (UKPDS) Group. Lancet 1998;352:854-65.

(57) Wulffele MG, Kooy A, de ZD, Stehouwer CD, Gansevoort RT. The effect of metformin on blood pressure, plasma cholesterol and triglycerides in type 2 diabetes mellitus: a systematic review. J Intern Med 2004;256:1-14.

(58) Cheang KI, Huszar JM, Best AM, Sharma S, Essah PA, Nestler JE. Long-term effect of metformin on metabolic parameters in the polycystic ovary syndrome. Diab Vasc Dis Res 2009;6:110-9.

\section{Significance}

Several groups, including Virmani's, have identified a novel macrophage subset that responds to intraplaque hemorrhage and has properties that may limit its atherogenic effects. We have termed these Mhem and previously implicated the transcription factor ATF1. This work shows that ATF1-mediated activation is initiated by AMPK in both human and murine macrophages responding to heme. This leads to coordinated induction of HO-1 and LXR, which link antioxidant protection with protection from lipid accumulation. The drug metformin initiates a closely similar pathway, which may explain its increased effect on preventing diabetic macrovascular disease. In vivo and human translational studies are warranted to follow these findings up. 
Figure 1

A

Heme $(10 \mu \mathrm{M})$

\begin{tabular}{|c|c|c|c|c|c|c|c|}
\hline Time (h) & T0 & 0.125 & 0.25 & 0.5 & 1 & 2 & 4 \\
\hline p-AMPK (60kDa) & 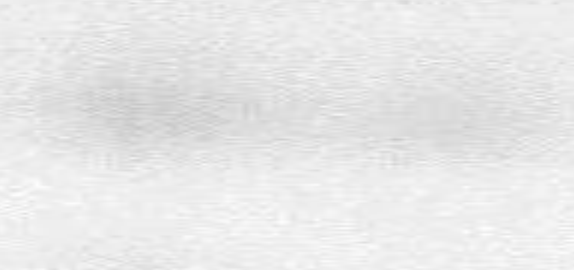 & $x_{2}=$ & 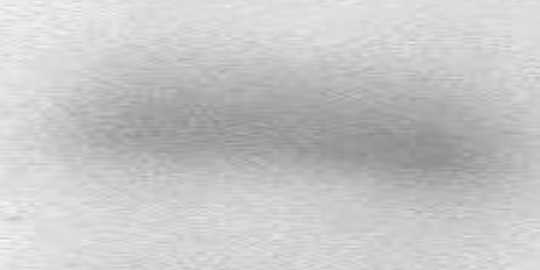 & $=0$ & $=$ & $=$ & as \\
\hline t-AMPK $(60 \mathrm{kDa})$ & 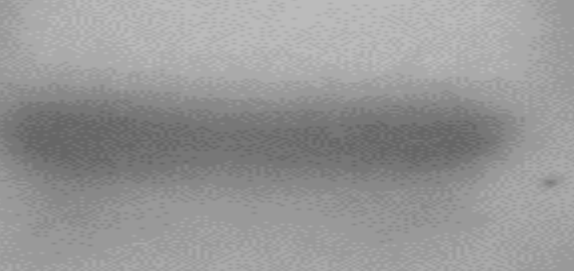 & - & 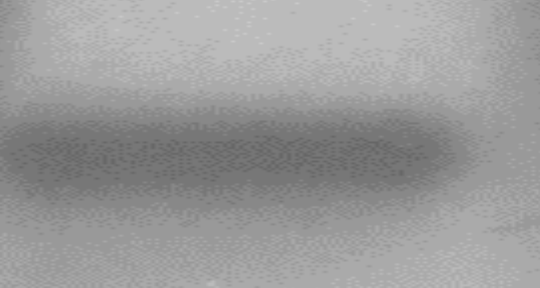 & $=$ & 3 & - & 5 \\
\hline p-ATF1 (37kDa) & & $\sin$ & -2 & 急 & & & Ex \\
\hline t-ATF1 (37kDa) & - & - & - & - & - & $=$ & $\infty$ \\
\hline HO-1 (27kDa) & & & & & $=0$ & $x^{2}=$ & $=$ \\
\hline LXR- $\beta$ (27kDa) & & & & & & & $\infty$ \\
\hline
\end{tabular}

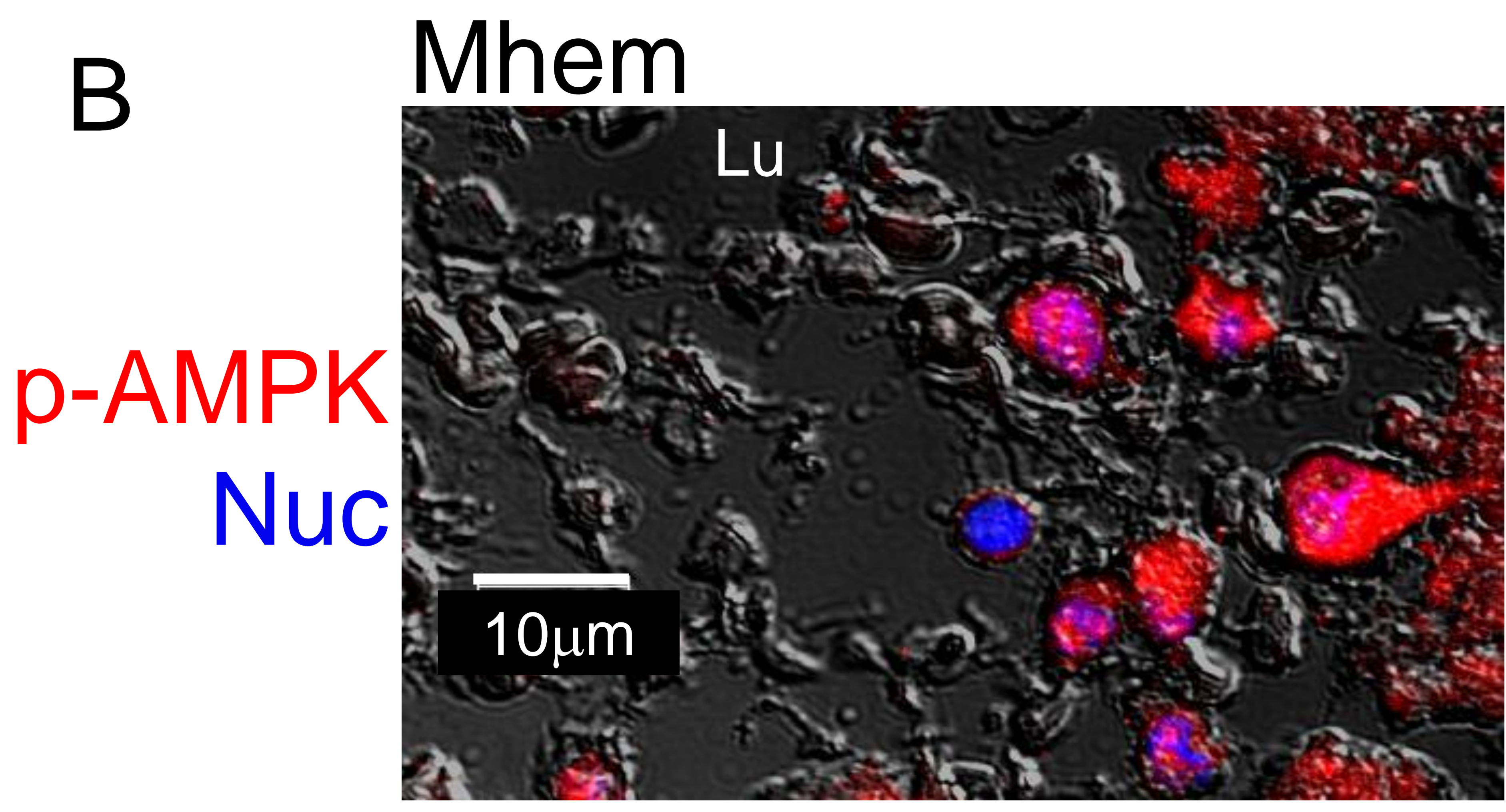

Foam cell

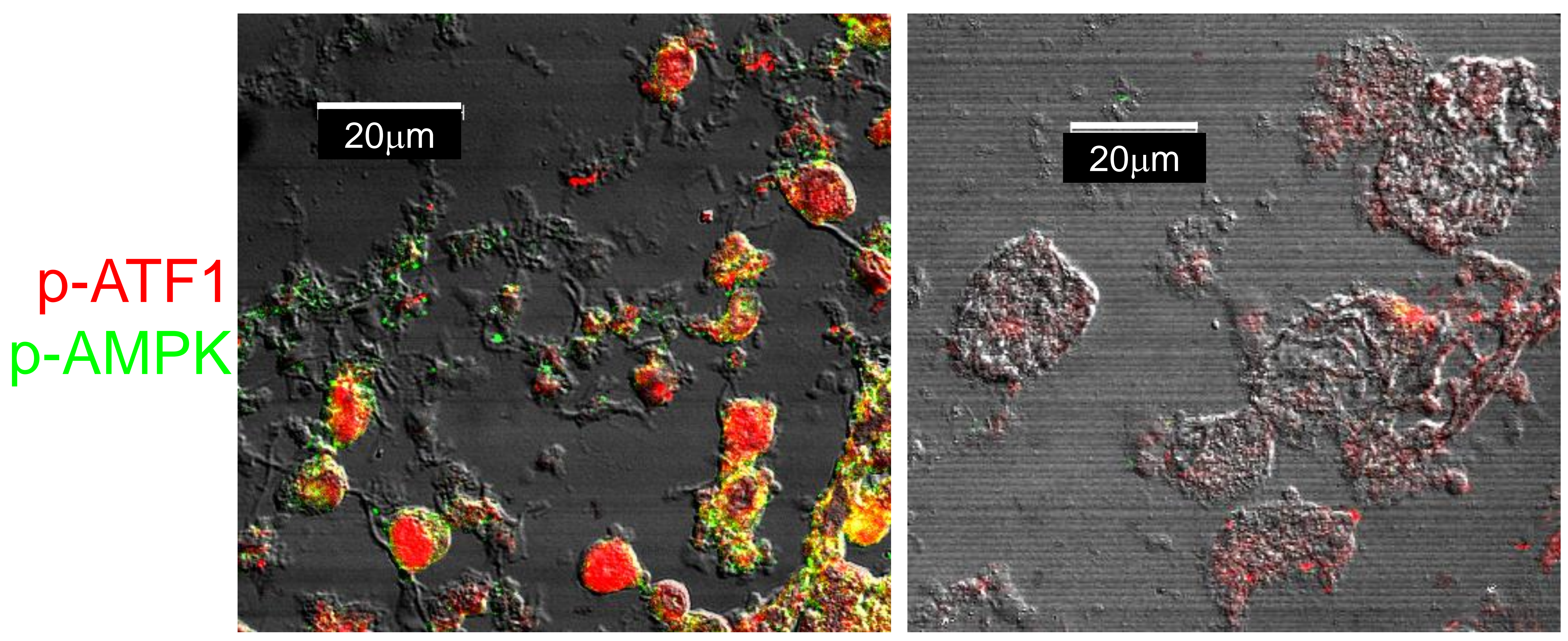


Figure 2

A

\begin{tabular}{|r|l|l|l|l|l|l|}
\hline Heme $(10 \mu \mathrm{M})$ & & & + & + & & \\
\hline Metformin $(10 \mu \mathrm{M})$ & & & & & + & + \\
\hline Dorsomorphin $(1 \mu \mathrm{M})$ & & + & & + & & + \\
\hline
\end{tabular}

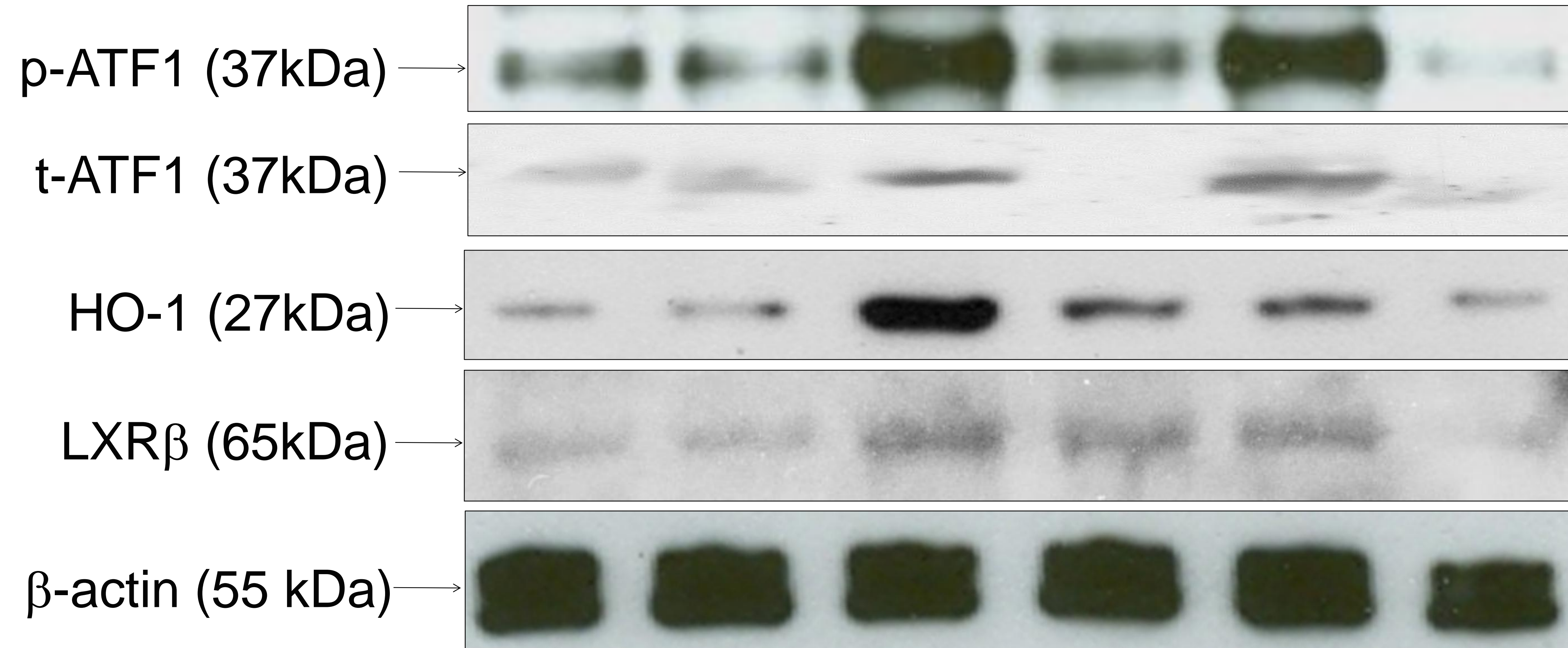

B
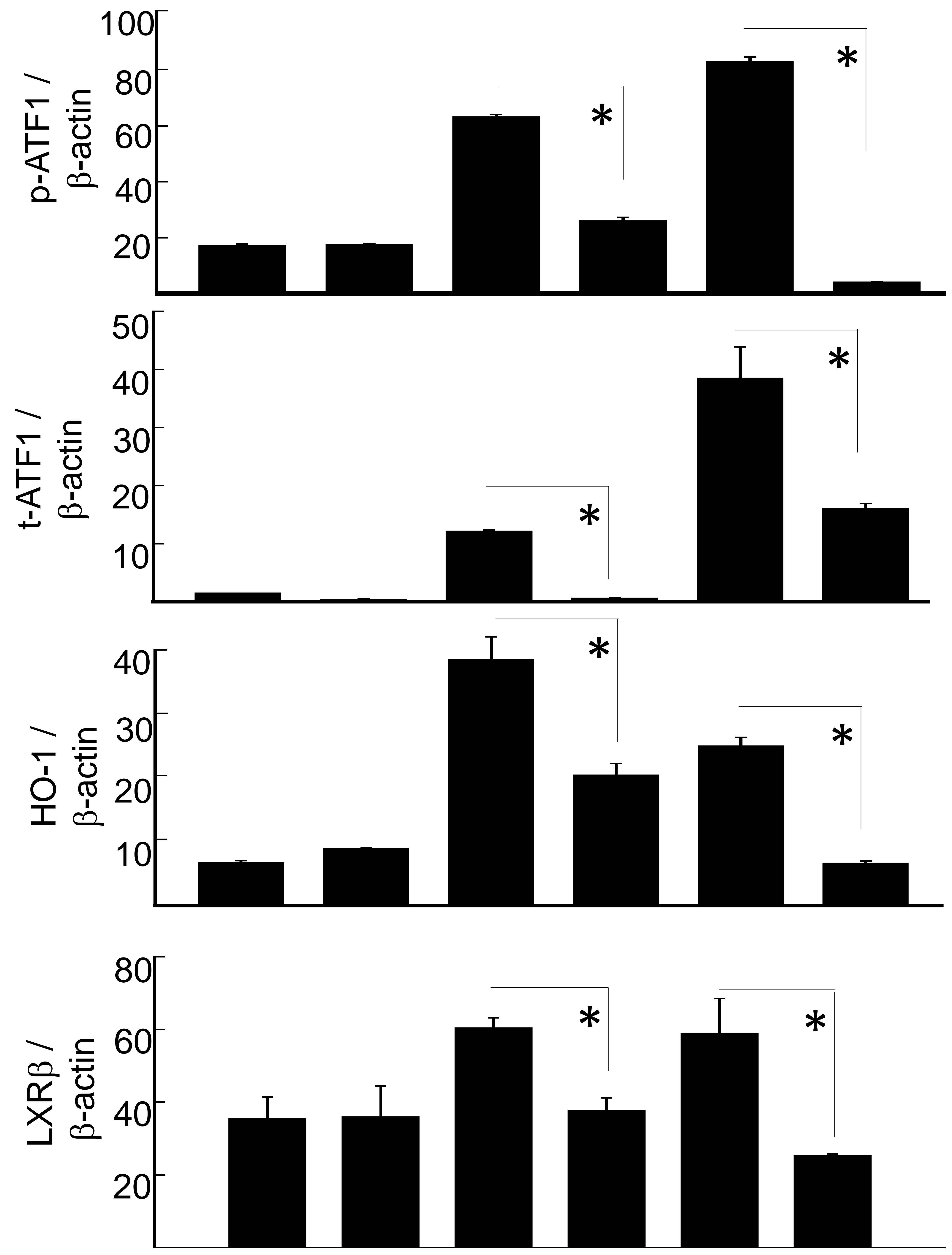


\section{Figure 3}

A

siRNA: $\quad$ Control-siRNA $\quad$ AMPK- $\alpha 1-$ siRNA Stimulant: Veh Heme Veh Heme

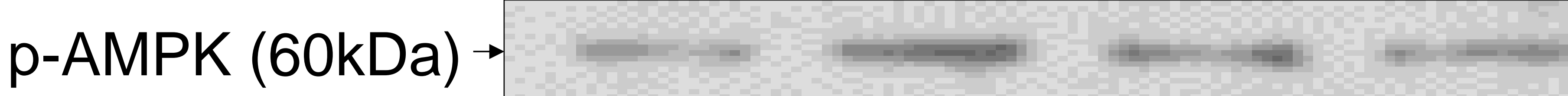
t-AMPK $(60 \mathrm{kDa})+$ p-ATF1 $(37 \mathrm{kDa}) \rightarrow$ t-ATF1 $(37 \mathrm{kDa}) \rightarrow$

$\mathrm{HO}-1(27 \mathrm{kDa}) \rightarrow$

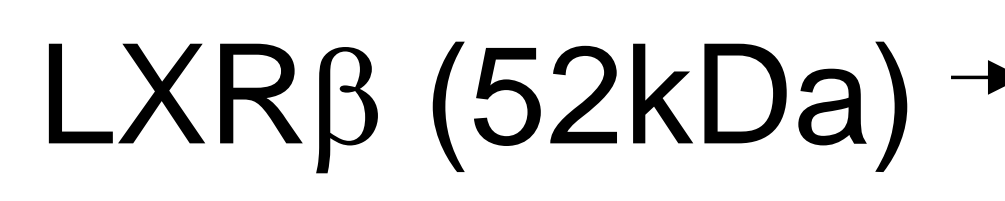

$\beta-\operatorname{actin}(55 \mathrm{kDa}) \rightarrow$
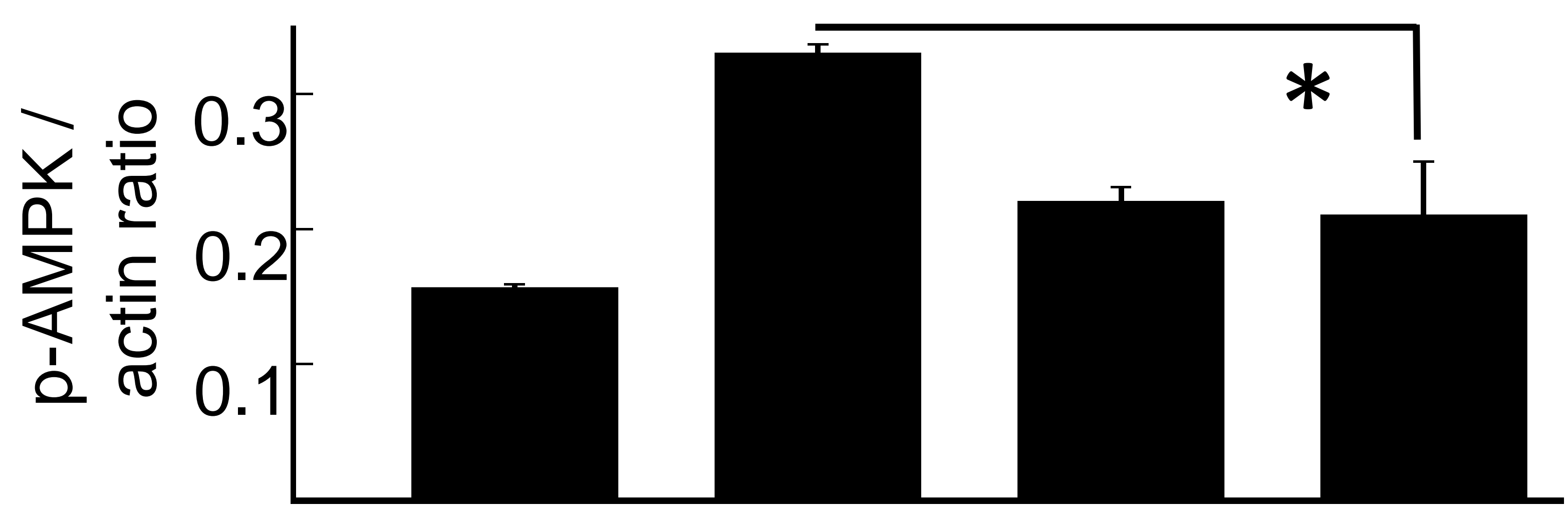

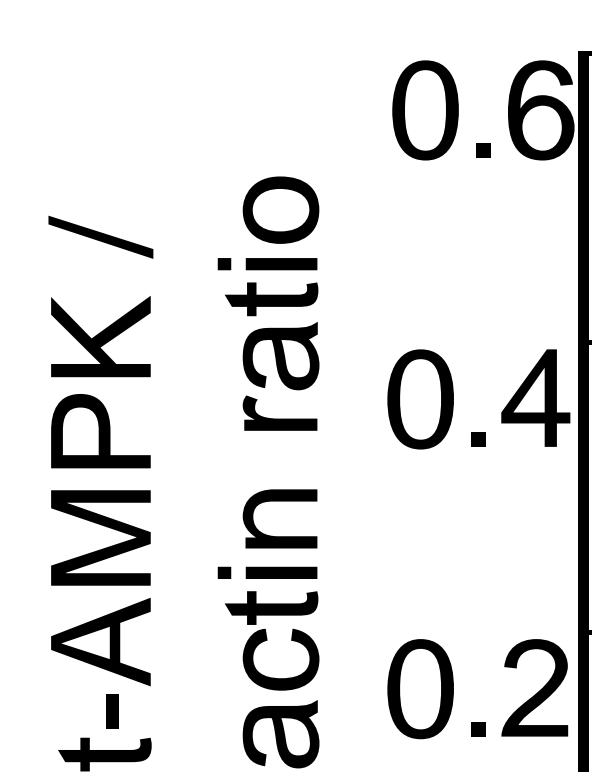

$*$
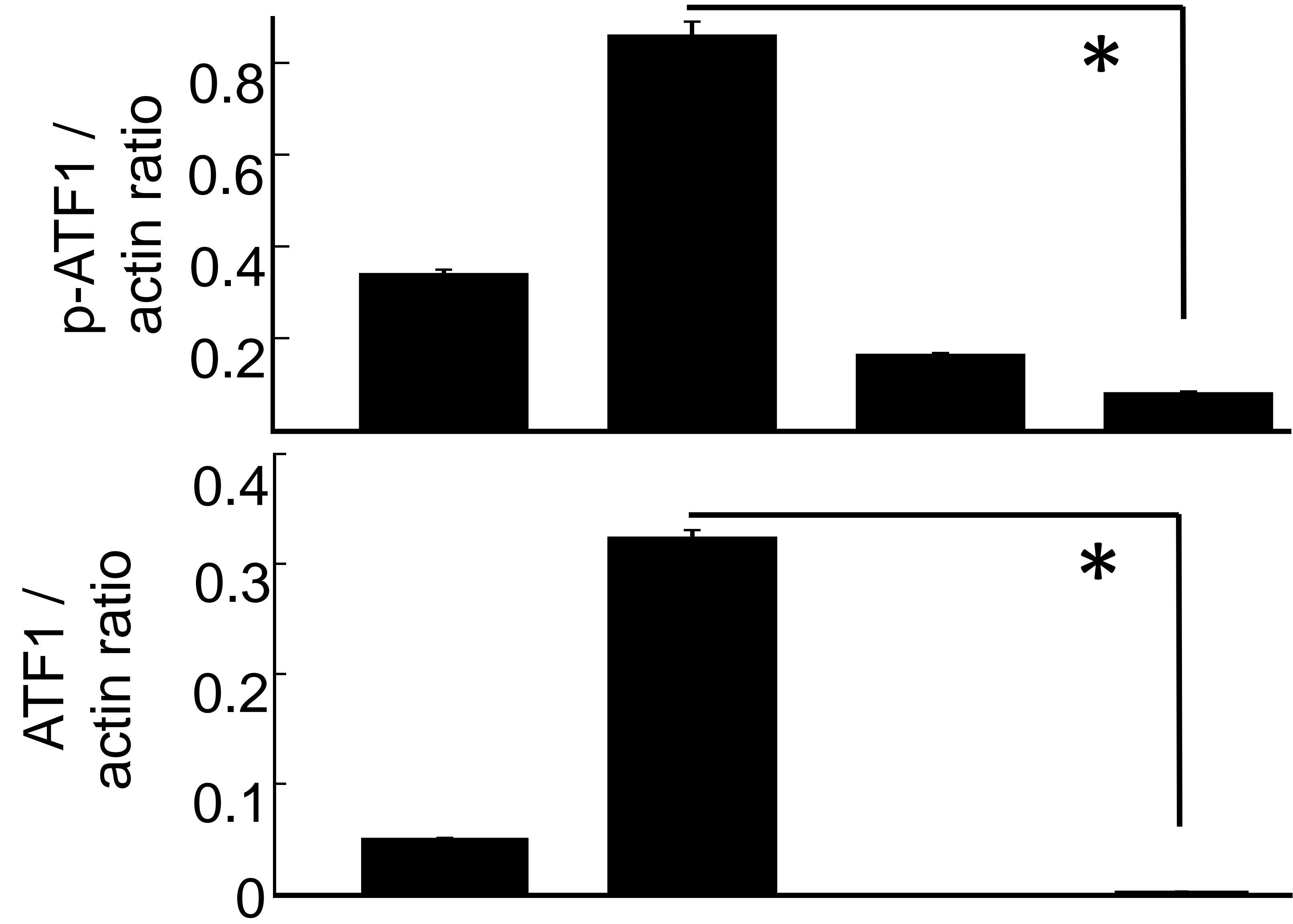

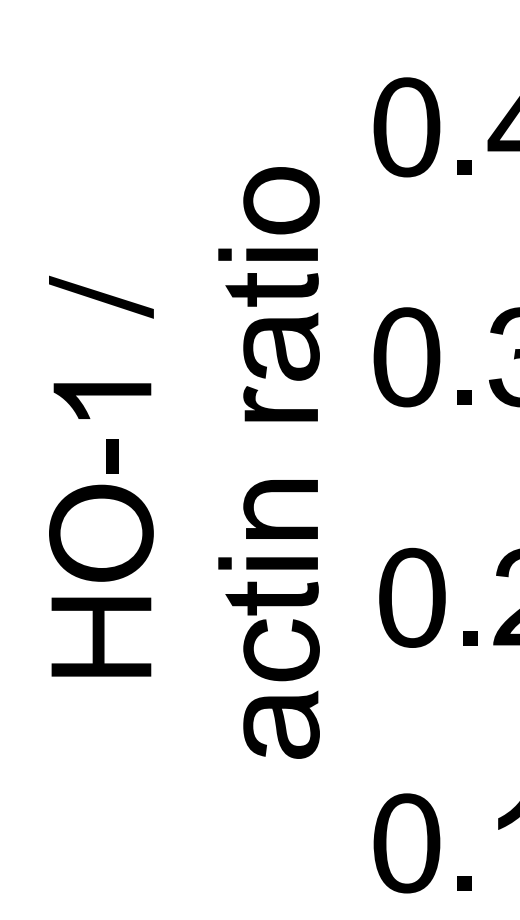

*

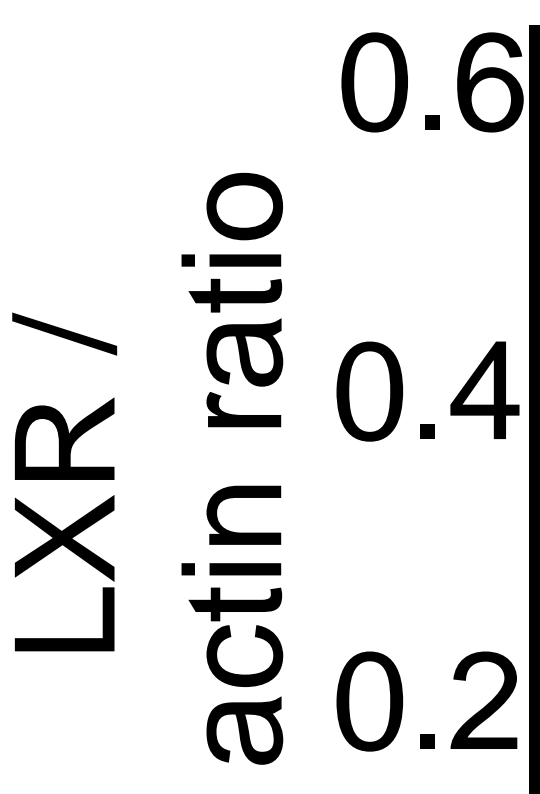
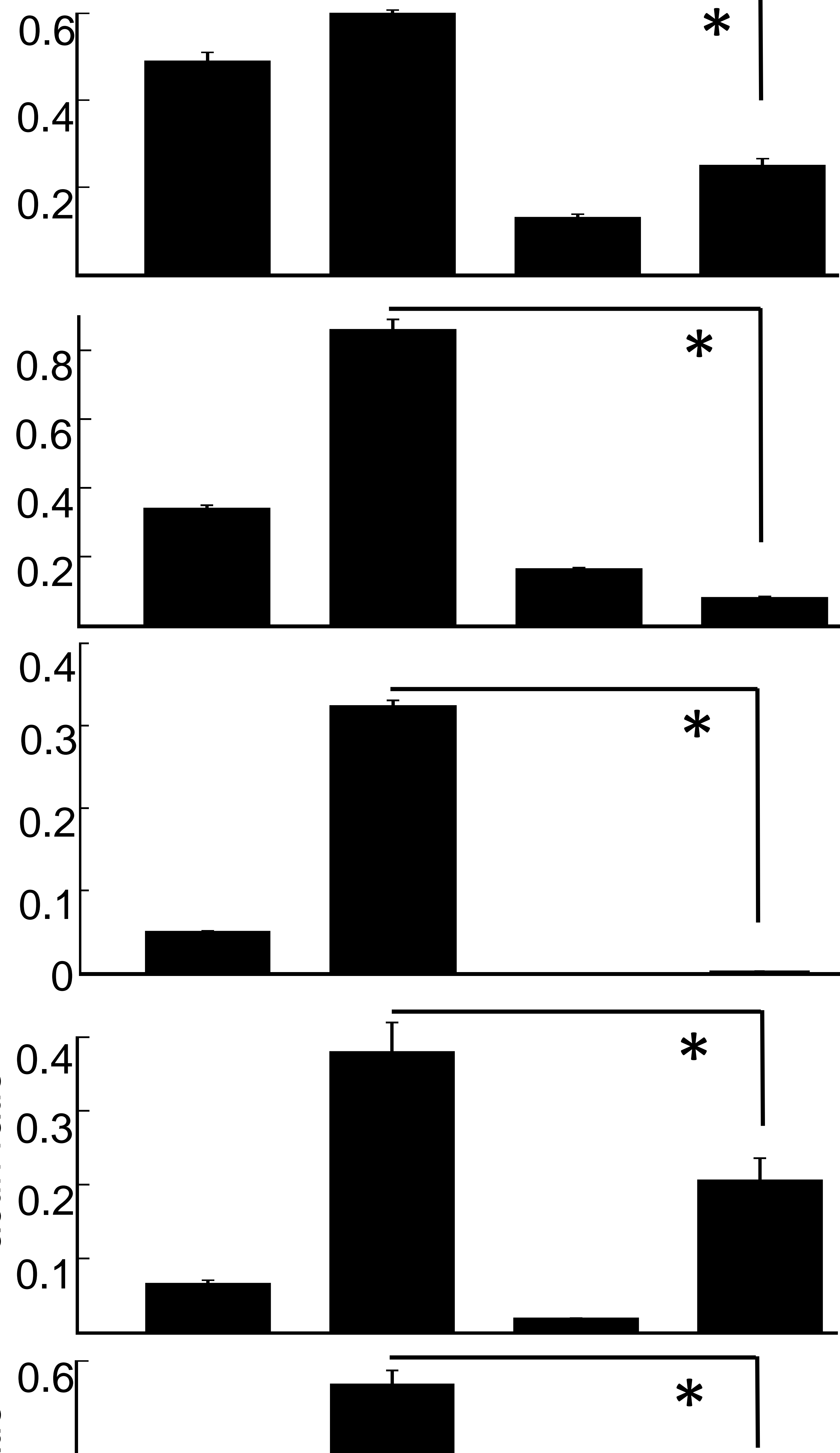

C

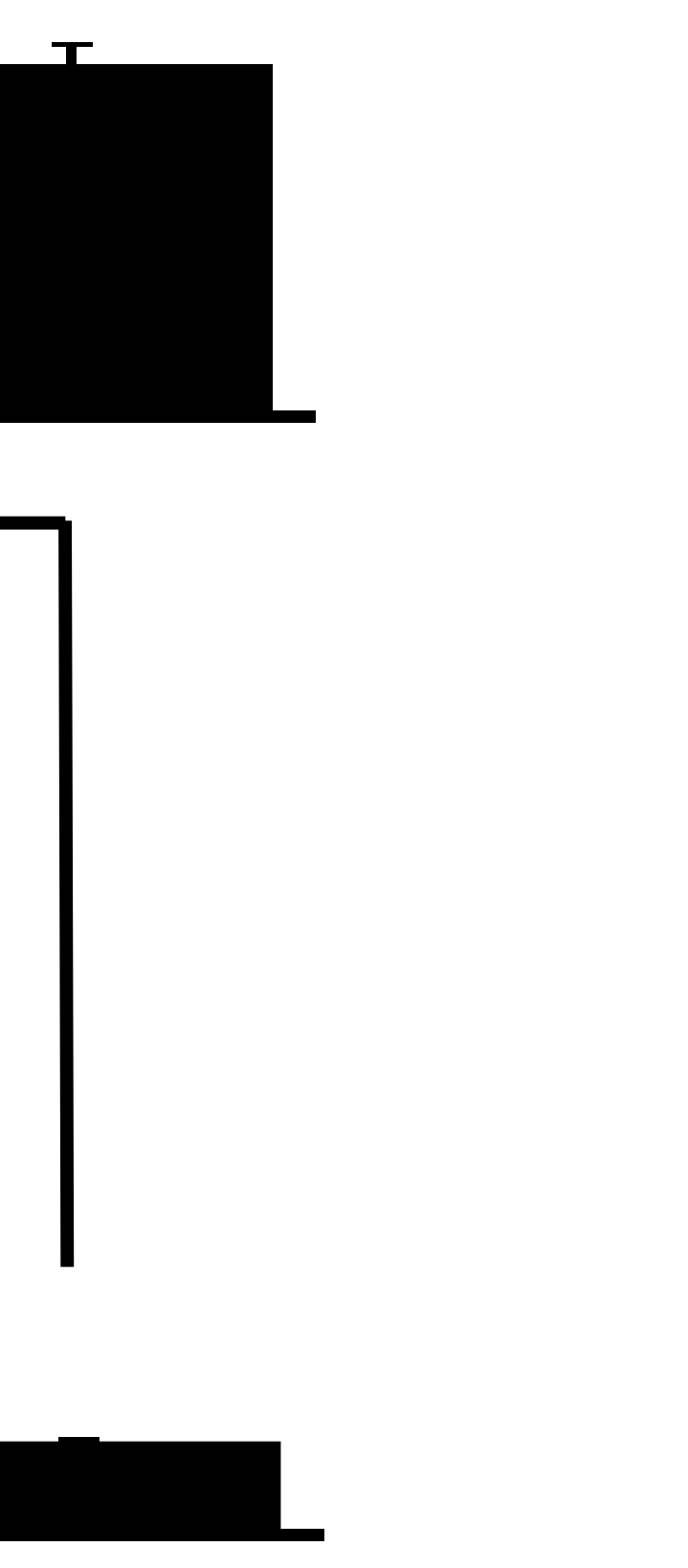


Figure 4

A
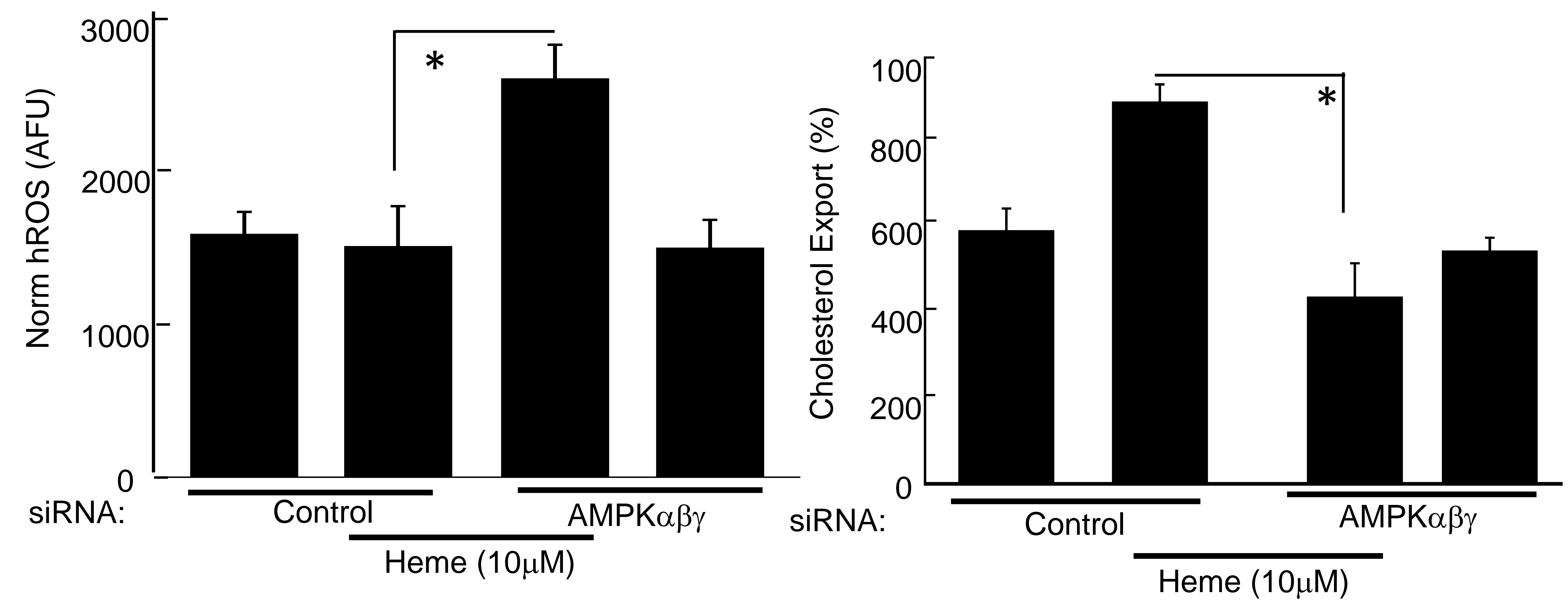

C

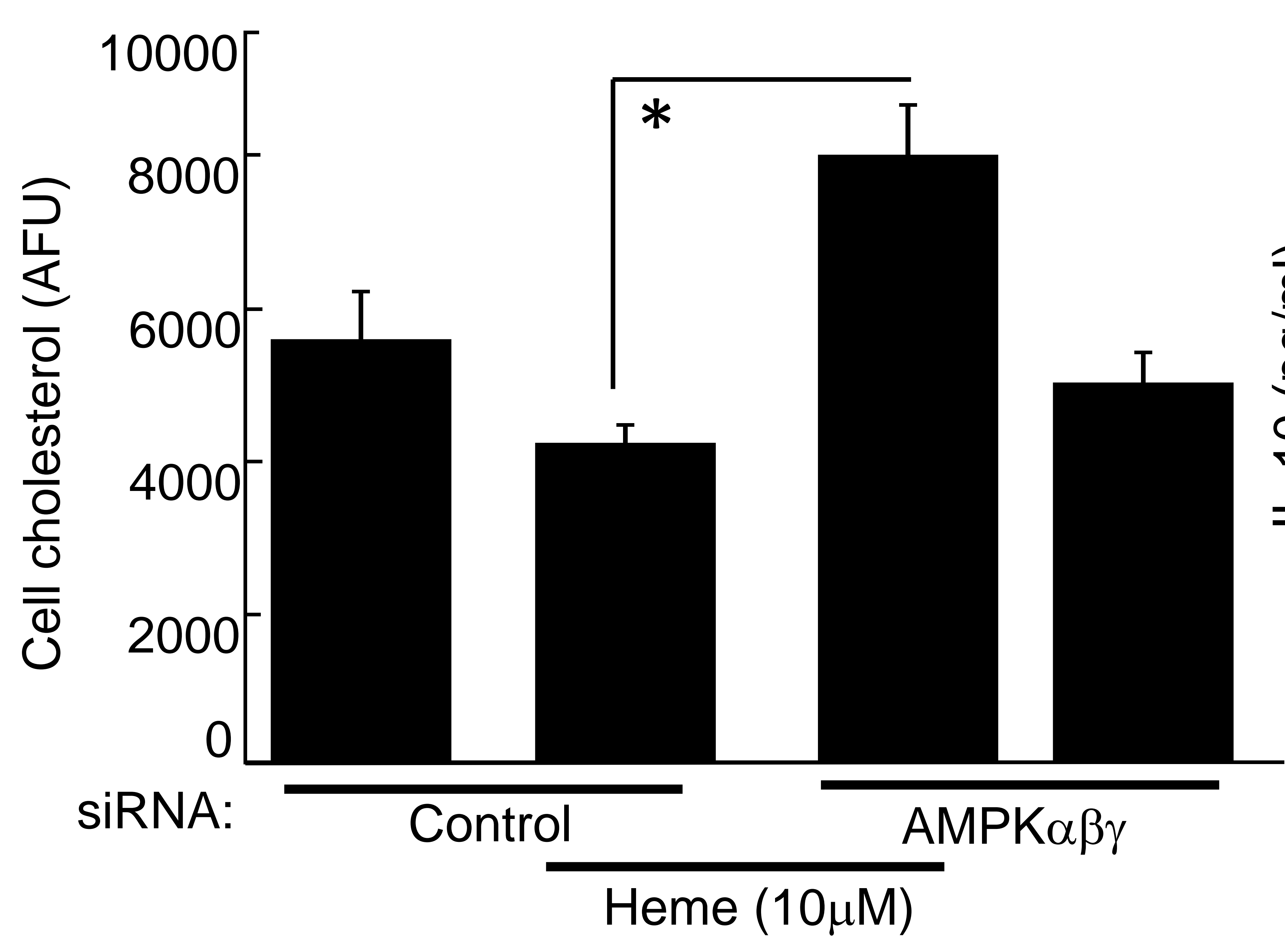

D

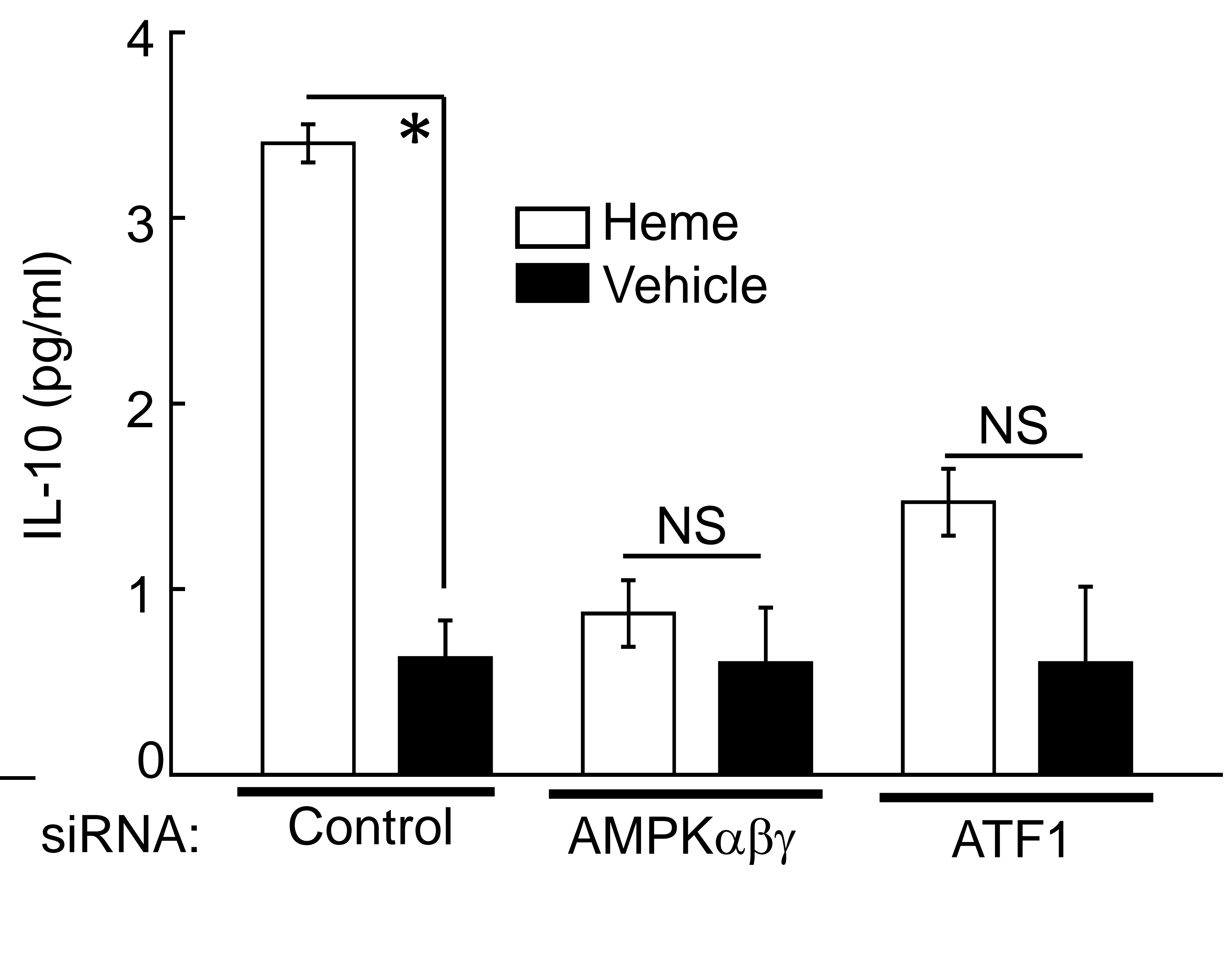

E

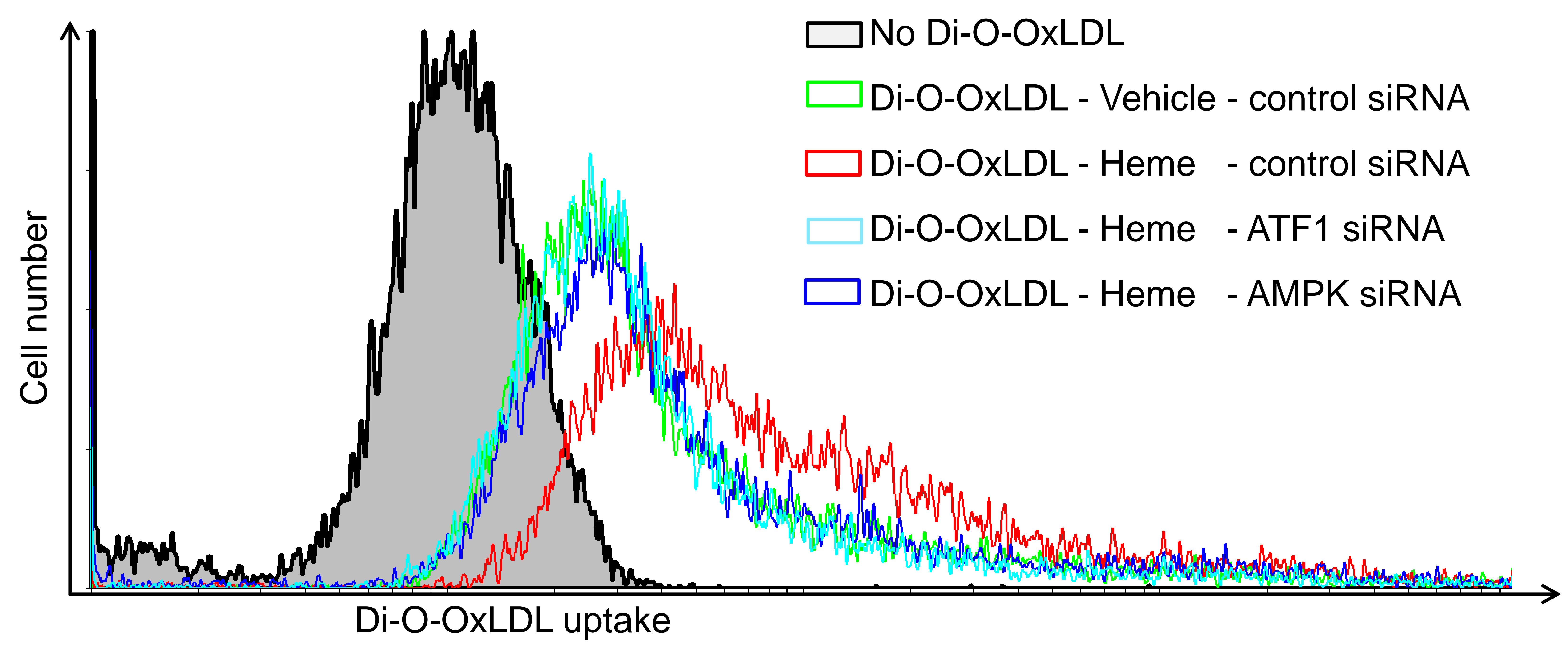




\section{Figure 5}
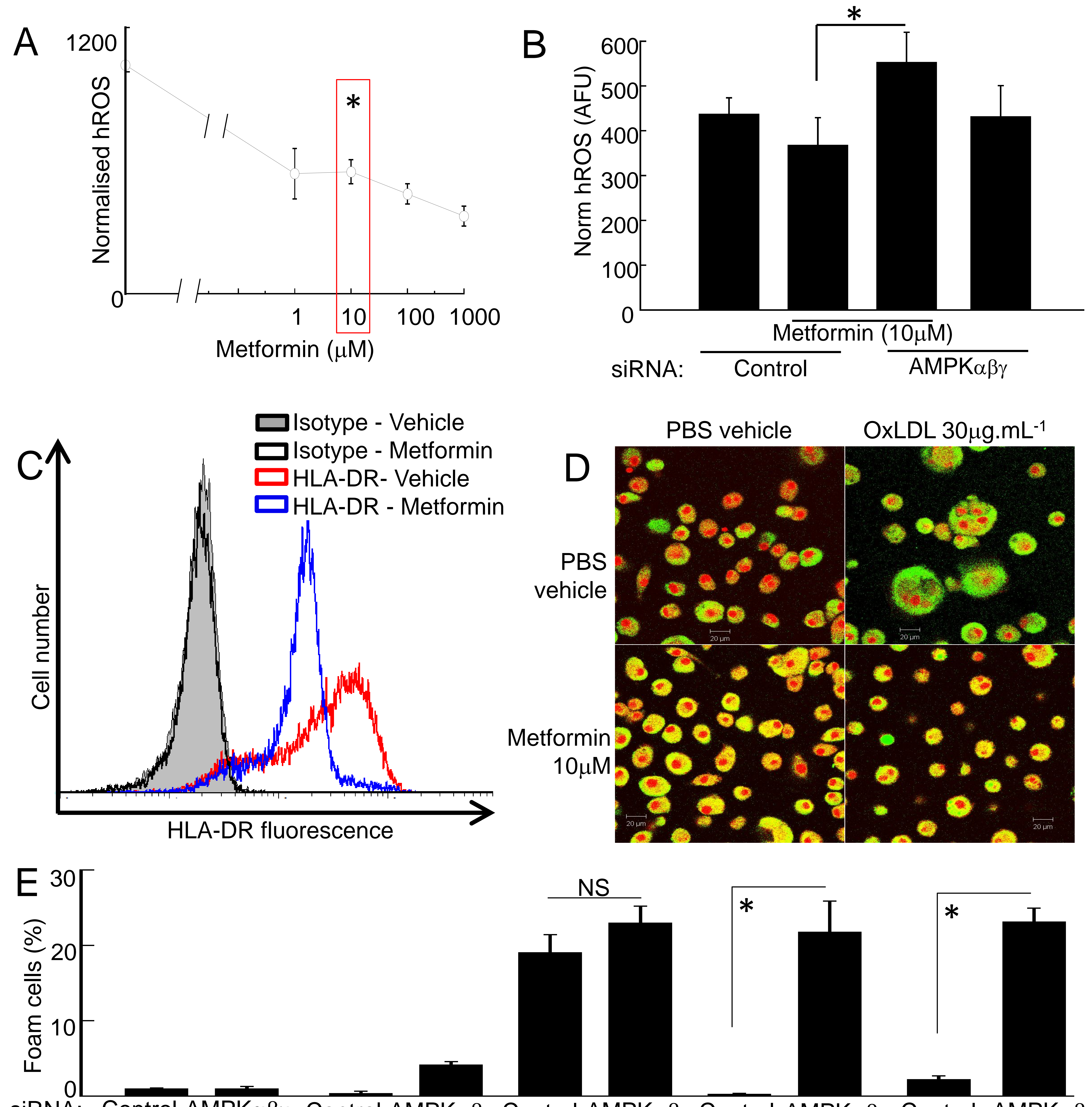

siRNA: Control AMPK $\alpha \beta \gamma$ Control AMPK $\alpha \beta \gamma$ Control AMPK $\alpha \beta \gamma$ Control AMPK $\alpha \beta \gamma$ Control AMPK $\alpha \beta \gamma$

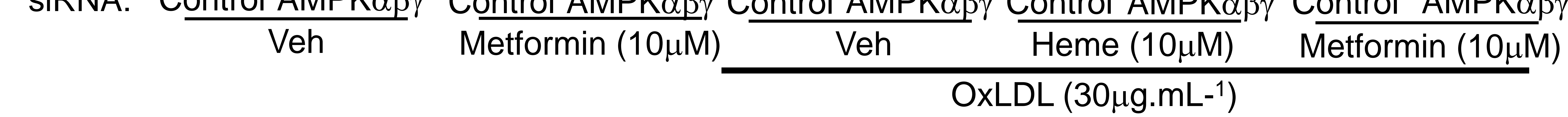
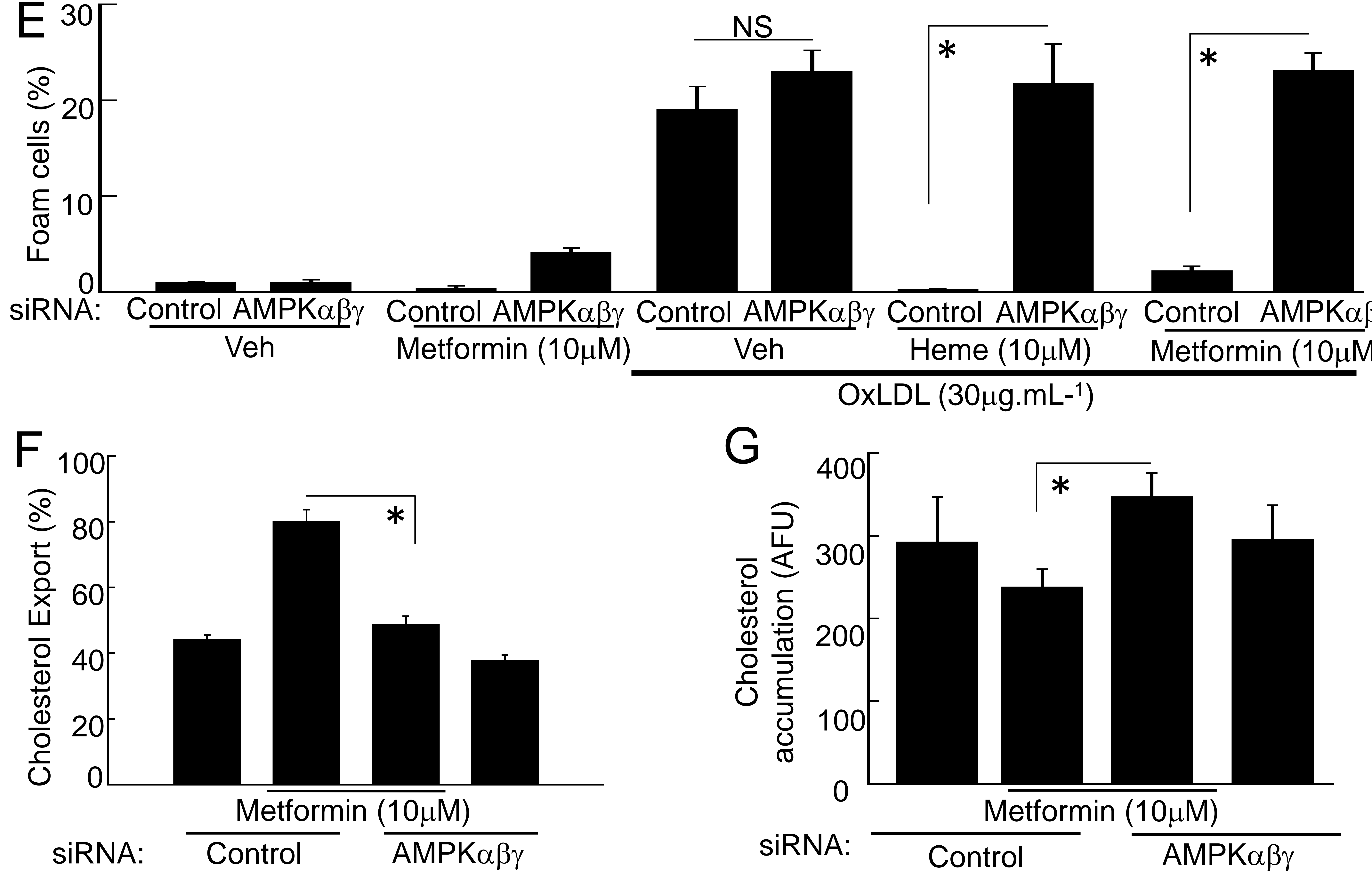
